\title{
Neutrino-nucleus reactions and nuclear structure
}

\author{
E Kolbe ${ }^{1}$, K Langanke ${ }^{2}$, G Martínez-Pinedo ${ }^{3,4}$ and P Vogel $^{5}$ \\ ${ }^{1}$ Department für Physik und Astronomie der Universität Basel, Basel, Switzerland \\ ${ }^{2}$ Institut for Fysik og Astronomi, Århus Universitet, DK-8000 Århus C, Denmark \\ ${ }^{3}$ Institut d'Estudis Espacials de Catalunya, Edifici Nexus, Gran Capità 2, E-08034 Barcelona, \\ Spain \\ ${ }^{4}$ Institució Catalana de Recerca i Estudis Avançats, Lluís Companys 23, E-08010 Barcelona, \\ Spain \\ 5 Department of Physics, California Institute of Technology, Pasadena, CA 91125, USA
}

Received 22 August 2003

Published 14 October 2003

Online at stacks.iop.org/JPhysG/29/2569

\begin{abstract}
The methods used in the evaluation of the neutrino-nucleus cross section are reviewed. Results are shown for a variety of targets of practical importance. Many of the described reactions are accessible in future experiments with neutrino sources from the pion and muon decays at rest, which might be available at the neutron spallation facilities. Detailed comparison between the experimental and theoretical results would establish benchmarks needed for verification and/or parameter adjustment of the nuclear models. Having a reliable tool for such calculation is of great importance in a variety of applications, e.g. the neutrino oscillation studies, detection of supernova neutrinos, description of the neutrino transport in supernovae and description of the r-process nucleosynthesis.
\end{abstract}

\section{Introduction}

There is now a convincing evidence that neutrinos are massive and the existence of neutrino oscillations has been convincingly demonstrated. This conclusion is based to a large extent on the observation of the neutrino induced reactions on complex nuclei, which also play essential roles in various aspects of nucleosynthesis as well as supernova collapse and supernova neutrino detection. Most of them have not been studied experimentally so far and their cross sections, which are needed in all applications, are typically based on nuclear theory. Spallation neutrino sources with their significant neutrino fluxes represent a unique opportunity to establish several benchmark measurements of the most significant neutrinonucleus reactions. These measurements, in turn, can be used to gauge the accuracy and reliability of the corresponding nuclear models.

In this work we review selected theoretical results of particular importance for neutrino detection, supernovae and nucleosynthesis. Theoretical description of the neutrino induced reactions is a challenging proposition, since the energy scales of interest span a vast region, 
from the few $\mathrm{MeV}$ for solar neutrinos, to tens of $\mathrm{MeV}$ for the interpretation of experiments with the muon and pion decay at rest and the detection of supernova neutrinos, to hundreds of $\mathrm{MeV}$ or few $\mathrm{GeV}$ for the detection atmospheric neutrinos. While reactions induced by low-energy neutrinos are sensitive to details of nuclear structure, $\mathrm{GeV}$ neutrinos, like other weak probes of similar energy, interact dominantly with individual nucleons in the nucleus, which can then be treated as an ensemble of non-interacting but bound protons and neutrons.

First, let us briefly review the general formalism adopted for the analysis of the charged current reactions,

$$
v_{e}+{ }_{Z} X_{N} \rightarrow{ }_{Z+1} X_{N-1}^{*}+\mathrm{e}^{-}
$$

and its analogues with $\bar{v}_{e}$ as well as with muon neutrinos and antineutrinos. The formalism can be easily modified for the neutral current reactions

$$
v+{ }_{Z} X_{N} \rightarrow{ }_{Z} X_{N}^{*}+v^{\prime}
$$

In the derivation of the relevant cross sections we follow the prescription given by Walecka [1] which is based on the standard current-current form for the weak interaction Hamiltonian governing these reactions. After a multipole expansion of the weak nuclear current and application of the extreme relativistic limit (final lepton energy $E_{\ell} \gg$ lepton mass $m_{\ell} c^{2}$ ) the neutrino (antineutrino) cross section for excitation of a discrete target state is given by $[1,2]$

$$
\left(\frac{\mathrm{d} \sigma_{i \rightarrow f}}{\mathrm{~d} \Omega_{\ell}}\right)_{\nu, \bar{v}}=\frac{\left(G_{F} V_{u d}\right)^{2} p_{\ell} E_{\ell}}{\pi} \frac{\cos ^{2} \frac{\Theta}{2}}{\left(2 J_{i}+1\right)} F\left(Z \pm 1, \epsilon_{\ell}\right)\left[\sum_{J=0}^{\infty} \sigma_{C L}^{J}+\sum_{J=1}^{\infty} \sigma_{T}^{J}\right]
$$

where

$$
\sigma_{C L}^{J}=\left|\left\langle J_{f}\left\|\tilde{M}_{J}(q)+\frac{\omega}{q} \tilde{L}_{J}(q)\right\| J_{i}\right\rangle\right|^{2}
$$

and

$$
\begin{aligned}
& \sigma_{T}^{J}=\left(-\frac{q_{\mu}^{2}}{2 q^{2}}\right.\left.+\tan ^{2} \frac{\Theta}{2}\right) \times\left[\left|\left\langle J_{f}\left\|\tilde{J}_{J}^{\mathrm{mag}}(q)\right\| J_{i}\right\rangle\right|^{2}+\left|\left\langle J_{f}\left\|\tilde{J}_{J}^{\mathrm{el}}(q)\right\| J_{i}\right\rangle\right|^{2}\right] \\
& \mp \tan \frac{\Theta}{2} \sqrt{\frac{-q_{\mu}^{2}}{q^{2}}+\tan ^{2} \frac{\Theta}{2}} \times\left[2 \operatorname{Re}\left\langle J_{f}\left\|\tilde{J}_{J}^{\mathrm{mag}}(q)\right\| J_{i}\right\rangle\left\langle J_{f}\left\|\tilde{J}_{J}^{\mathrm{el}}(q)\right\| J_{i}\right\rangle^{*}\right] .
\end{aligned}
$$

Here $\Theta$ is the angle between the incoming and outgoing leptons, and $q_{\mu}=(\omega, \vec{q})(q=|\vec{q}|)$ is the 4-momentum transfer. The minus-(plus) sign in equation (5) refers to the neutrino (antineutrino) cross section. The quantities $\tilde{M}_{J}, \tilde{L}_{J}, \tilde{J}_{J}^{\mathrm{el}}$ and $\tilde{J}_{J}^{\mathrm{mag}}$ denote the multipole operators for the charge, the longitudinal and the transverse electric and magnetic parts of the 4-current, respectively. Following [1,3] they can be written in terms of one-body operators in the nuclear many-body Hilbert space. The cross section involves the reduced matrix elements of these operators between the initial state $J_{i}$ and the final state $J_{f}$. (See $[4,5]$ for the slightly more complicated formula valid also for nonrelativistic final lepton energy.)

For low-energy electrons and positrons the Fermi function $F\left(Z, E_{\ell}\right)$ accounts for the Coulomb interaction between the final charged lepton and the residual nucleus in the chargedcurrent processes. We use the Coulomb correction derived by numerical solution of the Dirac equation for an extended nuclear charge [6]:

$$
F\left(Z, E_{\ell}\right)=F_{0}\left(Z, E_{\ell}\right) L_{0} \quad \text { with } \quad F_{0}\left(Z, E_{\ell}\right)=4\left(2 p_{l} R\right)^{2(\gamma-1)}\left|\frac{\Gamma(\gamma+\mathrm{i} y)}{\Gamma(2 \gamma+1)}\right|^{2} \mathrm{e}^{\pi y} .
$$


Here $Z$ denotes the atomic number of the residual nucleus in the final channel, $E_{\ell}$ the total lepton energy (in units of $m_{\ell} c^{2}$ ) and $p_{l}$ the lepton momentum (in units of $m_{\ell} c$ ), $R$ is the nuclear radius (in units of $\frac{\hbar}{m_{\ell} c}$ ) and $\gamma$ and $y$ are given by ( $\alpha=$ fine structure constant)

$$
\gamma=\sqrt{1-(\alpha Z)^{2}} \quad \text { and } \quad y=\alpha Z \frac{E_{\ell}}{p_{\ell}} .
$$

The numerical factor $L_{0}$ in (6), which describes the finite charge distribution and screening corrections, is nearly constant $(\approx 1.0)$, and can be well approximated by a weakly decreasing linear function in $p_{\ell}$.

At higher energies, and for muons at essentially all energies, the Fermi function valid for $s$-wave leptons is a poor approximation for the Coulomb effect since higher partial waves also contribute for $p_{\ell} R \geqslant 1$. Guided by the distorted-wave approximation of quasielastic electron scattering, we treat in that case the Coulomb effects in the 'effective momentum approximation' in which the outgoing lepton momentum $p_{\ell}$ is replaced by the effective momentum

$$
p_{\text {eff }}=\sqrt{E_{\text {eff }}^{2}-m_{\ell}^{2}} \quad E_{\text {eff }}=E_{\ell}-V_{C}(0),
$$

where $V_{C}(0)=3 e^{2} Z / 2 R$ is the Coulomb potential at the origin. In the work presented here, the Coulomb effect is taken into account not only by using the effective momentum, but also by replacing the phase-space factor $p_{\ell} E_{\ell}$ by $p_{\text {eff }} E_{\text {eff }}$ (see also [7] where this procedure is called modified effective momentum approximation, and shown to work quite well). In practice we use a smooth interpolation between these two regimes of treatment of the Coulomb effects.

We calculate the differential cross section (3) as a function of the initial neutrino energy $\epsilon_{\nu}$, the excitation energy of the nucleus $\omega$ and the scattering angle $\Theta$. The 3-momentum transfer $q \equiv|\vec{q}|$ is equal to

$$
q=\sqrt{\left(E_{v}-p_{\ell}\right)^{2}+4 E_{v} p_{\ell} \sin ^{2} \frac{\Theta}{2}} \simeq \sqrt{\omega^{2}+4 E_{v}\left(E_{v}-\omega\right) \sin ^{2} \frac{\Theta}{2}},
$$

where the last expression is valid in the relativistic limit $\left(E_{\ell} \gg m_{\ell}\right)$ for the final lepton. The total cross section is obtained from the differential cross sections by summing (or integrating) over all possible final nuclear states and by numerical integration over the angles.

As explicitly used in the derivation of the cross section formula above, in neutrino-induced reactions the nucleus is excited by multipole operators $O_{\lambda}$ which scale like $(q R / \hbar c)^{\lambda}$, where $R$ is the nuclear radius $\left(R \sim 1.2 A^{1 / 3} \mathrm{fm}\right)$. As the momentum transfer is of the order of the neutrino energy $E_{v}$, neutrino-nucleus reactions involve multipole operators with successively higher rank $\lambda$ with increasing neutrino energy. Since the nuclear Hamiltonian does not commute with $O_{\lambda}$, the response of the operator is fragmented over many nuclear states. However, for each multipole most of the strength resides in a collective excitation, the giant resonance, with a width of a few $\mathrm{MeV}$. The centroids of the giant resonances of the various multipoles grow in energy with increasing rank, roughly like $\lambda \hbar \omega$, where $\hbar \omega \simeq 41 / A^{1 / 3} \mathrm{MeV}$ is a typical energy shell splitting in the nucleus. Furthermore, since the phase space is proportional to $p_{\ell} E_{\ell}$ the higher outgoing lepton energies are preferred. This suggests that the average nuclear excitation energy $\bar{\omega}$ lags behind the increasing neutrino energy, i.e., for sufficiently large neutrino energies the inequality $\bar{\omega}<E_{v} \simeq E_{\ell}$ holds. As a consequence, for neutrino energies significantly larger than the energies of the corresponding giant resonances, the neutrino-induced cross sections will depend on the total strength of the multipole excitation and its centroid energy, but will be less sensitive to its detailed energy distribution. Finally, at very high neutrino energies the neutrino will see the nucleus as an ensemble of independent bound nucleons and will interact with individual nucleons. In that regime we will consider only the quasielastic channel in which the struck nucleon is ejected. This is the channel most widely used in neutrino detectors. 
From these general considerations we can identify three different energy ranges with quite different demands on the details with which the nuclear structure should be treated:

(i) For relatively low neutrino energies, comparable to the nuclear excitation energy, neutrino-nucleus reactions are very sensitive to the appropriate description of the nuclear response. Thus, low-energy neutrino scattering requires a nuclear model which reproduces the important correlations among nucleons. The model of choice is the nuclear shell model, which accounts for nucleon-nucleon correlations via an effective interaction within a fixed model space for the valence nucleons. Nowadays, complete diagonalization for the lowest states in medium-mass nuclei (up to $A \sim 60$ ) is achievable in complete $0 \hbar \omega$ model spaces, i.e., considering all configurations of the valence nucleons in a full harmonic oscillator shell. For lighter nuclei, such as ${ }^{16} \mathrm{O}$, complete diagonalization can be performed in larger model spaces ( $4 \hbar \omega$ for ${ }^{16} \mathrm{O}, 6 \hbar \omega$ for ${ }^{12} \mathrm{C}$ ). Importantly, the shell model calculations have been proved to indeed reproduce the allowed (Fermi, Gamow-Teller) response for those nuclei for which diagonalizations can be performed in sufficiently large model spaces. For the lighter nuclei, where multi-shell calculations can be performed, the shell model also nicely describes forbidden transitions. An overview of recent shell model developments and applications is given in [8].

(ii) The random phase approximation (RPA) has been developed to describe the collective excitation of a nucleus by considering the one-particle one-hole excitations of the correlated ground state. In the standard RPA, all excited states are treated as bound states, leading to a discrete excitation spectrum. In the continuum RPA (CRPA) (see e.g. $[9,10]$ and references therein) the final states have the appropriate scattering asymptotics for energies above the nucleon-emission thresholds; consequently the excitation spectrum in the CRPA is continuous. The RPA or CRPA are the methods of choice at intermediate neutrino energies where the neutrino reactions are sensitive dominantly to the total strength and the energy centroids of the giant resonances.

(iii) At high incoming energies neutrinos, like other electroweak probes, scatter 'quasi-freely' on individual nucleons. The remaining nucleons can be treated as (non-interacting) spectators. This situation is realized in the Fermi gas model [11] where a full relativistic treatment of the hadronic weak vertex is included. The Fermi motion and the binding energy are characterized by parameters that can be determined from electron scattering experiments. In this approximation the expansion in multipoles is no longer necessary. The nuclear form factors for the quasi-free scattering are expressed in terms of the single nucleon matrix elements which depend only on the 4-momentum transfer $q^{2}$ and on the nuclear momentum distribution.

Above we pointed out the important role of collective excitations. The centroid position of these excitations deviates noticeably from the independent particle estimate of $\lambda \hbar \omega$ due to the residual particle-hole interaction. Thus, empirical evidence or nuclear structure calculation is needed to determine their energy. On the other hand, the total strength is often fixed by sum rules in an essentially model independent way. Well-known example of such sum rules is the Ikeda sum rule for the Gamow-Teller strength

$$
\sum_{i} B(G T ; Z \rightarrow Z+1)_{i}-\sum_{i} B(G T ; Z \rightarrow Z-1)_{i}=3(N-Z),
$$

or the Thomas-Reiche-Kuhn sum rule for the dipole strength

$$
\sum_{i}\left(E_{i}-E_{0}\right) B(E 1 ; 0 \rightarrow i)=\frac{9}{4 \pi} \frac{\hbar^{2}}{2 M_{p}} \frac{N Z}{A} e^{2} .
$$


Even though both of these sum rules could be violated to some extent (e.g., when the internal structure of the nucleons is not properly treated), the dependence on the neutron and proton numbers $N$ and $Z$ remains valid. Note that the Ikeda sum rule involves the difference of the strengths. However, in nuclei with neutron excess, $N>Z$, the second term, the total strength $B(G T ; Z \rightarrow Z-1)$ is much smaller than the first one, and so the sum rule determines the strength in the $(p, n)$ channel. In symmetric nuclei with $N=Z$ the Ikeda sum rule, unfortunately, does not help in fixing the Gamow-Teller strength.

To calculate the various partial neutrino-induced reaction cross sections for neutrinoinduced reactions we assume a two-step process. In the first step we calculate the charged current $\left(v_{l}, l^{-}\right)$and $\left(\bar{v}_{l}, l^{+}\right)$cross sections (where $l=e$ or $\mu$ ), or the neutral current cross section $\left(\nu, v^{\prime}\right)$ as a function of excitation energy in the final nucleus. These calculations are performed within the RPA or CRPA and considering all multipole operators up to a certain $J$ and both parities. In the second step one calculates for each final state with well-defined energy the branching ratios into the various decay channels using the statistical model code SMOKER [12]. As possible final states in the residual nucleus the SMOKER code considers the experimentally known levels supplemented at higher energies by an appropriate level density formula [12]. Proton, neutron, $\alpha$ and $\gamma$ emission are included in the code as decay channels. If the decay leads to an excited level of the residual nucleus, the branching ratios for the decay of this state are calculated in an analogous fashion [13]. Keeping track of the energies of the ejected particles and photons during the cascade, and weighting them with appropriate branching ratios and the corresponding primary charged- or neutral-current cross sections, we determine the various partial particle emission cross sections.

\section{Comparison of different methods}

Here we demonstrate, using the neutrino interaction with ${ }^{16} \mathrm{O}$ as an illustration, how different theoretical methods can be used at different neutrino energies. We show that at certain transition energy intervals the corresponding methods give essentially identical results.

\subsection{Shell model versus CRPA}

We first consider the description of the charged current reactions on ${ }^{16} \mathrm{O}$ at relatively low energies. The reaction thresholds are $15.4 \mathrm{MeV}$ for the ${ }^{16} \mathrm{O}\left(v_{e}, \mathrm{e}^{-}\right)^{16} \mathrm{~F}$ (which is unbound) and $11.4 \mathrm{MeV}$ for the ${ }^{16} \mathrm{O}\left(\bar{v}_{e}, \mathrm{e}^{+}\right){ }^{16} \mathrm{~N}$ reaction.

The shell model evaluation of the cross sections was performed back in 1987 by Haxton [14]. In that work the low lying positive parity states were described in a full $2 \hbar \omega$ shell model. The transitions to negative parity states were described using the effective density matrices, scaled to describe measured form factors from electron scattering.

The shell model results can be compared with the CRPA. The CRPA calculations used the finite range residual force based on the Bonn potential, and all multipole operators with $J \leqslant 9$ and both parities were included. The free nucleon form factors were used, with no quenching. The procedure was tested by evaluating the total muon capture rates (dominated by the negative parity multipoles) for ${ }^{12} \mathrm{C},{ }^{16} \mathrm{O}$ and ${ }^{40} \mathrm{Ca}$ [19], as well as the partial capture rates to the bound $0^{-}, 1^{-}$and the $2^{-}$ground state in ${ }^{16} \mathrm{~N}[19]^{6}$. Good agreement with these muon capture rates tests the method at momentum transfer $q \sim m_{\mu} \sim 100 \mathrm{MeV}$.

We compare the cross sections evaluated by the two methods in figure 1, where we show the cross sections evaluated in both methods and averaged over the Fermi-Dirac distribution

6 The partial rates listed in table 2 of [19] should be multiplied by a factor 1000 . 


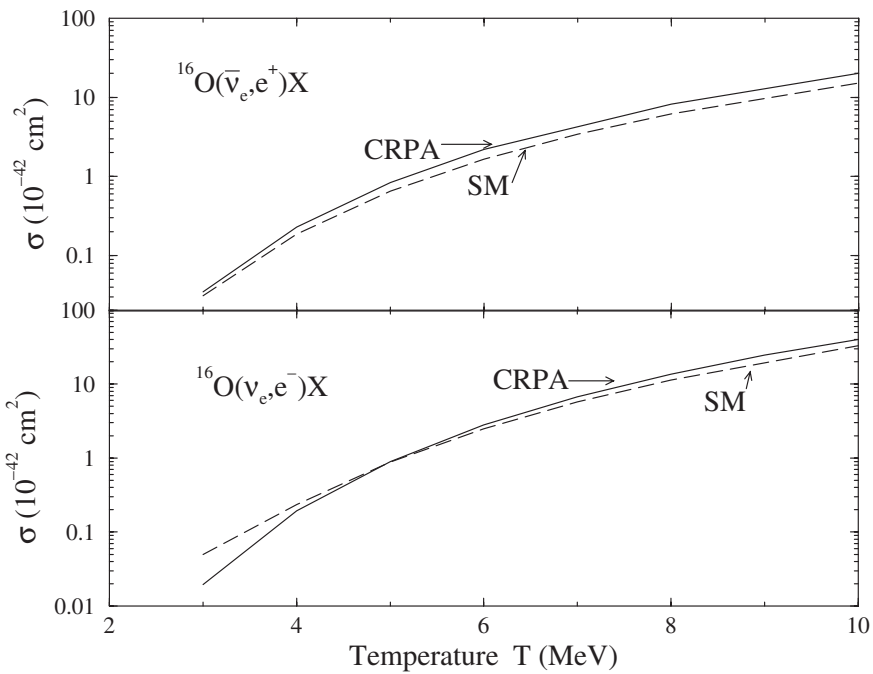

Figure 1. Comparison of the CRPA (full lines) and shell model (dashed lines) cross sections. The upper panel is for the $\bar{v}_{e}$ induced reaction and the lower one is for the reaction induced by $v_{e}$.

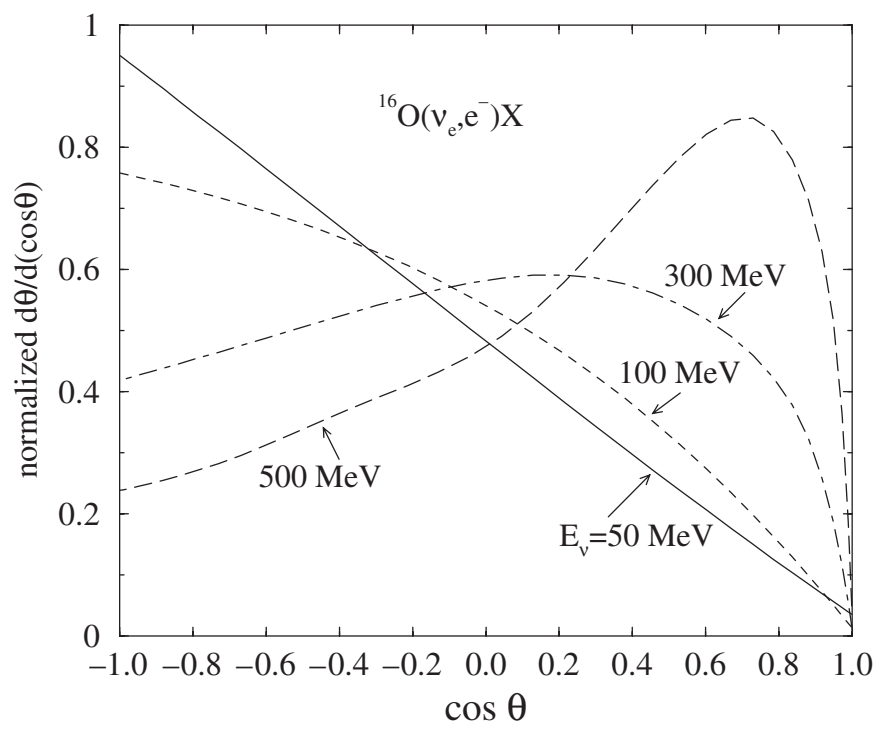

Figure 2. The CRPA angular distributions.

corresponding to the temperature $T$ and vanishing chemical potential. The agreement is excellent suggesting that both methods are capable of describing the weak reaction rates in this energy regime, provided that they can be successfully tested on relevant quantities, such as the muon capture rates, nuclear photoabsorption cross section, or inelastic electron scattering leading to the states populated by the weak processes.

The angular distribution of the emitted electrons with respect to the incoming neutrino beam is shown in figure 2. Note the electron emission is predominantly in the backward direction at low energies (also obtained in the nuclear shell model), but it gradually changes 

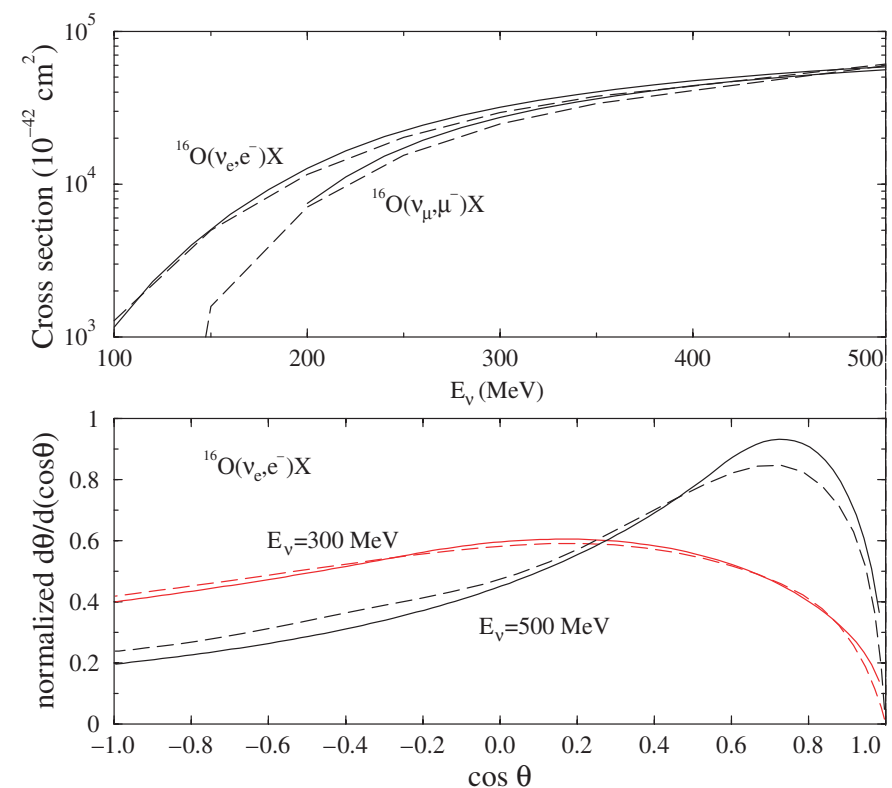

Figure 3. Comparison of the relativistic Fermi gas model (full lines) and the CRPA (dashed lines). The parameters of the Fermi gas model were $p_{f}=225 \mathrm{MeV}$ and $e_{b}=27 \mathrm{MeV}$. The cross sections are shown in the upper panel for the two indicated reactions. The angular distributions for $E_{v}=$ 300 and $500 \mathrm{MeV}$ are shown in the lower panel. The CRPA results are shown with full lines, RFG with dashed lines.

to the forward one at higher energies. Thus, for $E_{v} \geqslant 500 \mathrm{MeV}$ the direction of the electron can be used to determine the direction of the incoming neutrino.

We have thus identified the energy region, somewhere near about $50 \mathrm{MeV}$ of neutrino energy, where the two discussed methods, the nuclear shell model and the CRPA, give essentially identical results. For lower energies the nuclear shell model is the method of choice. As the energy increases, the shell model calculations become increasingly difficult. The number of states increases rapidly, and the effective interaction to be used becomes more uncertain. However, as we argued above, at higher neutrino energies, above, say, $E_{v} \geqslant$ $100 \mathrm{MeV}$, the details of the nuclear correlations become less important and what matters are the positions and strengths of giant resonances. The CRPA is capable of describing these quantities and thus, in our opinion, it is the method of choice at the intermediate neutrino energy range, approximately $100 \mathrm{MeV} \leqslant E_{v} \leqslant 500 \mathrm{MeV}$. As the energy increases further, the CRPA calculations become computationally more difficult (more multipoles and higher nuclear excitation energies must be included). At the same time, the nuclear response, at least for the quasielastic regime, becomes simpler.

\subsection{CRPA versus relativistic Fermi gas model}

The CRPA and relativistic Fermi gas model (RFG) methods are compared in figure 3 and agree remarkably well in both the total quasielastic cross section and the angular distribution of the outgoing electrons. The latter is particularly important, because the zenith angle distribution of the atmospheric neutrinos is based on the assumption that one can deduce the incoming neutrino direction (and hence its flightpath) from the direction of the observed charged lepton. Our CRPA calculations confirm that, indeed, below about $500 \mathrm{MeV}$ of neutrino energy the 
emitted electron (or muon) is essentially uncorrelated with the direction of the incoming neutrino. Above these energies, the emitted lepton moves dominantly in the direction of the incoming neutrino, hence one can, statistically, correlate the two. This tendency, naturally, becomes more pronounced at higher $E_{v}$ values.

Let us stress that no attempt was made to adjust the Fermi gas model parameters to obtain the agreement demonstrated in figure 3 ; these are just standard values of the Fermi momentum $p_{f}$ and of the parameter $e_{b}$ which characterizes the average nucleon binding energy.

Thus, we conclude that at energies $E_{v} \simeq 300 \mathrm{MeV}$ one can safely switch from the CRPA to the simpler Fermi gas model description of the quasielastic charged current reactions. However, it should be stressed that at low energies there are important checks in the form of muon capture rates as well as (for ${ }^{12} \mathrm{C}$ ) neutrino induced reaction for the neutrino beams from the muon and pion decay at rest. Similar tests do not exist, or were at least less exploited, for $\sim 1 \mathrm{GeV}$ neutrino energies.

\section{Neutrino ${ }^{12} \mathrm{C}$ interaction}

The nucleus ${ }^{12} \mathrm{C}$ is particularly important for the study of the neutrino-nucleus scattering. Liquid scintillator detectors, for example KARMEN and LSND, contain hydrogen and ${ }^{12} \mathrm{C}$ nuclei. Therefore, a number of experimental results exist in this case as byproducts of the neutrino oscillation searches performed with these detectors.

The measurements include charged-current reactions induced by both electron- $[16,17]$ and muon-neutrinos [17], exciting both the ground and continuum states in ${ }^{12} \mathrm{~N}$. As discussed below, the inclusive cross section for ${ }^{12} \mathrm{C}\left(v_{e}, e\right){ }^{12} \mathrm{~N}^{*}$ with the $v_{e}$ from the muon decay at rest (DAR) [16-18] agrees well with calculations, while in contrast, there is a discrepancy between calculations [19, 20, 22, 23] (with some notable exceptions [24, 25]) and the measured [17] inclusive cross section for ${ }^{12} \mathrm{C}\left(v_{\mu}, \mu\right){ }^{12} \mathrm{~N}^{*}$, which uses higher energy neutrinos from pion decay-in-flight (DIF). The disagreement is somewhat disturbing in light of the simplicity of the reaction and in view of the fact that parameter-free calculations, such as those in [19-21], describe well other weak processes governed by the same weak current nuclear matrix elements. Moreover, as shown in the following subsection, the exclusive reactions populating the ground state of the final nucleus, ${ }^{12} \mathrm{C}\left(v_{e}, e\right){ }^{12} \mathrm{~N}_{\mathrm{gs}}$ and ${ }^{12} \mathrm{C}\left(v_{\mu}, \mu\right){ }^{12} \mathrm{~N}_{\mathrm{gs}}$, and the neutral current reaction ${ }^{12} \mathrm{C}\left(v_{e}, v_{e}^{\prime}\right){ }^{12} \mathrm{C}(15.11 \mathrm{MeV})$ have been measured $[16,17]$ as well, and agree well with theoretical expectations.

\subsection{Exclusive reactions}

Among the states in the final nucleus ${ }^{12} \mathrm{~N}$, which is populated by the charged current reactions with beams of $v_{e}$ or $v_{\mu}$, the ground state $I^{\pi}=1^{+}$plays a special role. It is the only bound state in ${ }^{12} \mathrm{~N}$, and can be recognized by its positron decay $\left(T_{1 / 2}=11 \mathrm{~ms}\right)$ back to ${ }^{12} \mathrm{C}$. Moreover, the analogue of the ${ }^{12} \mathrm{~N}_{\mathrm{gs}}$, the $I^{\pi}=1^{+}$state with isospin $T=1$ at $15.11 \mathrm{MeV}$ in ${ }^{12} \mathrm{C}$, can be populated by the neutral current neutrino scattering, and is recognizable by its emission of the 15.11 MeV photon. Finally, even though there are several bound states in ${ }^{12} \mathrm{~B}$, its ground state, the analogue of the other two $\left(I^{\pi}, T\right)=\left(1^{+}, 1\right)$ states, is the state most strongly populated in muon capture on ${ }^{12} \mathrm{C}$. Again, the population of the bound states in ${ }^{12} \mathrm{~B}$ can be separated from the continuum by observing its electron decay $\left(T_{1 / 2}=20.2 \mathrm{~ms}\right)$.

Theoretical evaluation of the exclusive cross sections is constrained by the obvious requirement that the same method, and the same parameters, must also describe the related processes, i.e. the positron decay of ${ }^{12} \mathrm{~N}$, the $\beta$ decay of ${ }^{12} \mathrm{~B}$, the $M 1$ strength of the $15.11 \mathrm{MeV}$ state in ${ }^{12} \mathrm{C}$, and the partial muon capture rate leading to the ground state of ${ }^{12} \mathrm{~B}$. It turns out 
Table 1. Comparison of calculated and measured cross sections, in units of $10^{-42} \mathrm{~cm}^{-2}$ and averaged over the corresponding neutrino spectra, for the neutrino induced transitions ${ }^{12} \mathrm{C}_{\mathrm{gs}} \rightarrow{ }^{12} \mathrm{~N}_{\mathrm{gs}}$ and ${ }^{12} \mathrm{C}_{\mathrm{gs}} \rightarrow{ }^{12} \mathrm{C}(15.11 \mathrm{MeV})$. For the decay at rest the $v_{e}$ spectrum is normalized from $E_{v}=0$, while for the decay in flight the $v_{\mu}$ and $\bar{v}_{\mu}$ spectra are normalized from the corresponding threshold. See the text for explanations.

\begin{tabular}{lcll}
\hline & $\begin{array}{l}{ }^{12} \mathrm{C}\left(v_{e}, \mathrm{e}^{-}\right){ }^{12} \mathrm{~N}_{\mathrm{gs}} \\
\text { decay at rest }\end{array}$ & $\begin{array}{l}{ }^{12} \mathrm{C}\left(v_{\mu}, \mu^{-}\right){ }^{12} \mathrm{~N}_{\mathrm{gs}} \\
\text { decay in flight }\end{array}$ & $\begin{array}{l}{ }^{12} \mathrm{C}\left(v, v^{\prime}\right){ }^{12} \mathrm{C}(15.11) \\
\text { decay at rest }\end{array}$ \\
\hline Experiment [16] & $9.4 \pm 0.5 \pm 0.8$ & - & $11 \pm 0.85 \pm 1.0$ \\
Experiment [17] & $9.1 \pm 0.4 \pm 0.9$ & $66 \pm 10 \pm 10$ & - \\
Experiment [18] & $10.5 \pm 1.0 \pm 1.0$ & - & - \\
Shell model [26] & 9.1 & 63.5 & 9.8 \\
CRPA [19, 20] & 8.9 & 63.0 & 10.5 \\
EPT [27] & 9.2 & 59 & 9.9 \\
\hline
\end{tabular}

that this requirement essentially determines the neutrino induced cross section for the energies of present interest. It does not matter which method of calculation is used, as long as the constraints are obeyed.

The comparison between the measured and calculated values is shown in table 1. There, three rather different methods of calculation were used, all giving excellent agreement with the data.

The first approach is a restricted shell-model calculation. Assuming that all structures in the considered low-lying states are generated by the valence nucleons in the $p$-shell, and that the two-body currents (pion-exchange currents) are negligible, there are only four one-body densities (OBD) which fully describe all necessary nuclear matrix elements. In this case, it is necessary to use the one-body densities chosen (ad hoc) in such a way that all the auxiliary data mentioned above are correctly reproduced. This then gives the results listed in line 4 of table 1 .

Effects of configurations beyond the $p$ shell might explain the need for the renormalization of the one-body densities produced by a reasonable $p$-shell Hamiltonian. Therefore, the rates of all the reactions are also evaluated in the random phase approximation (RPA), which does include multishell correlations, while treating the configuration mixing within the $p$ shell only crudely. Again an adjustment is needed (a 'quenching' of all matrix elements by an universal, but substantial, factor 0.515 ). However, the neutrino cross sections in line 5 of table 1 agree with the measurements very well.

The third approach is the 'elementary-particle treatment' (EPT). Instead of describing nuclei in terms of nucleons, the EPT considers them elementary and describes transition matrix elements in terms of nuclear form factors deduced from experimental data. The EPT approach was extended in [26] to the higher neutrino energies relevant to the LSND decay-in-flight $v_{\mu}$ by appropriately including the lepton mass.

An example of the energy dependence of the exclusive cross section is shown in figure 4 for the $v_{\mu}$ induced exclusive reaction. As one can see, the cross section raises sharply from its threshold $\left(E_{\mathrm{thr}}=123 \mathrm{MeV}\right)$ and soon reaches its saturation value, i.e., it becomes almost energy independent. This means that the yield of the ${ }^{12} \mathrm{C}+v_{\mu}$ reaction essentially measures just the flux normalization above the reaction threshold. At the same time, the yield is insensitive to the energy distribution of the muon neutrinos in the beam.

\subsection{Inclusive reactions}

The inclusive reactions ${ }^{12} \mathrm{C}\left(v_{e}, e\right){ }^{12} \mathrm{~N}^{*}$, with $v_{e}$ neutrinos from the muon decay-at-rest and ${ }^{12} \mathrm{C}\left(v_{\mu}, \mu\right){ }^{12} \mathrm{~N}^{*}$ with the higher energy $v_{\mu}$ neutrinos from the pion decay-in-flight populate not 


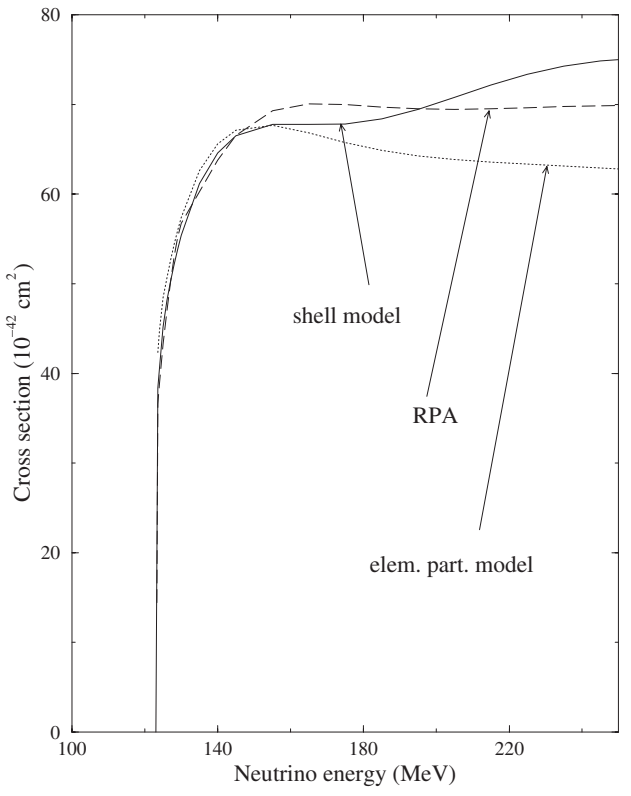

Figure 4. Energy dependence of the cross section for the reaction ${ }^{12} \mathrm{C}+v_{\mu} \rightarrow{ }^{12} \mathrm{~N}_{\mathrm{gs}}+\mu^{-}$.

only the ground state of ${ }^{12} \mathrm{~N}$ but also the continuum states. The corresponding cross sections involve folding over the incoming neutrino spectra and integrating over the excitation energies in the final nucleus. By convention, we shall use the term 'inclusive' for the cross section populating only the continuum (i.e., without the exclusive channel) for ${ }^{12} \mathrm{C}\left(v_{e}, e\right){ }^{12} \mathrm{~N}^{*}$ with the decay-at-rest $v_{e}$, while for the reaction ${ }^{12} \mathrm{C}\left(v_{\mu}, \mu\right){ }^{12} \mathrm{~N}^{*}$ with the decay-in-flight $v_{\mu}$ the term is used for the total cross section (the exclusive channel then represents only a small fraction of the total).

Muon capture, ${ }^{12} \mathrm{C}\left(\mu, v_{\mu}\right){ }^{12} \mathrm{~B}^{*}$, belongs also to this category. It involves momentum transfer of $q \approx m_{\mu}$, intermediate between the two neutrino capture reactions above. Since ${ }^{12} \mathrm{~B}$ and ${ }^{12} \mathrm{~N}$ are mirror nuclei, all three reactions should be considered together. In this case again the term 'inclusive' will be used only for the part of the rate populating the continuum in ${ }^{12} \mathrm{~B}$.

Which theoretical approach should one use in order to describe such reactions? One possibility is to use the continuum random phase approximation (CRPA). The method has been used successfully in the evaluation of the nuclear response to weak and electromagnetic probes [9]. In particular, it was tested, with good agreement, in the calculation of the inelastic electron scattering [28] on ${ }^{12} \mathrm{C}$ involving very similar excitation energies and momentum transfers as the weak processes of interest. As an example figure 5 shows the comparison of the experimental data and the results of the CRPA for the inclusive electron scattering [29]. One can see that the CRPA describes quite well both the magnitude and shape of this cross section over the entire range of excitation energies and momentum transfers.

For muon capture the CRPA [19] gives the inclusive rates of 0.342, 0.969 and $26.2 \times$ $10^{5} \mathrm{~s}^{-1}$ for ${ }^{12} \mathrm{C},{ }^{16} \mathrm{O}$ and ${ }^{40} \mathrm{Ca}$; to be compared with the measured rates of $0.320,0.924$ and $25.6 \times 10^{5} \mathrm{~s}^{-1}$ for the same nuclei. This good agreement is again obtained without any parameter adjustment. In particular, as discussed in [19], no renormalization of the axial vector coupling constant $g_{A}$ in nuclear medium is required. 


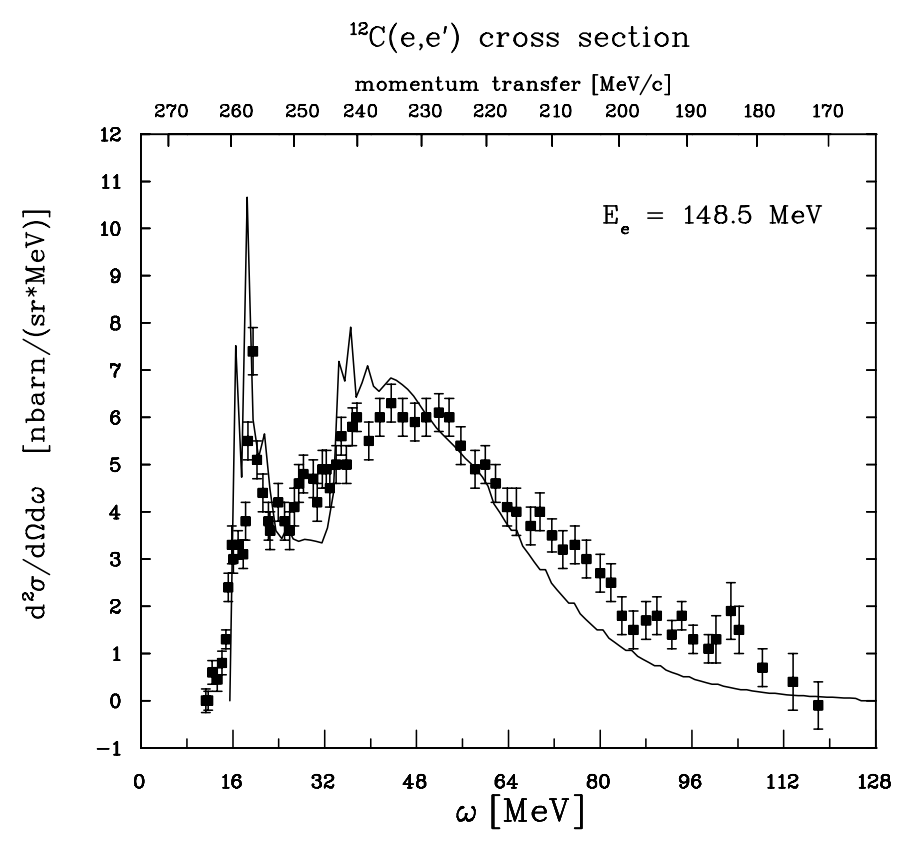

Figure 5. Data (points with error bars) and calculated cross section for the inclusive electron scattering on ${ }^{12} \mathrm{C}$ as a function of the excitation energy $\omega$. The corresponding momentum transfer is displayed on the upper scale.

What are the momentum transfers and excitation energies involved in the inclusive reactions which we would like to describe? For the ${ }^{12} \mathrm{C}\left(v_{e}, e\right){ }^{12} \mathrm{~N}^{*}$ with the electron neutrinos originating in the muon decay at rest, the typical momentum transfer is $\langle|\vec{q}|\rangle \simeq 50 \mathrm{MeV}$, and the typical excitation energy is $\omega \simeq 20 \mathrm{MeV}$. For the inclusive muon capture ${ }^{12} \mathrm{C}\left(\mu^{-}, v_{\mu}\right)^{12} \mathrm{~B}^{*}$ we have $\langle|\vec{q}|\rangle \simeq 90 \mathrm{MeV}$ and the typical excitation energy is $\omega \simeq 25 \mathrm{MeV}$. Finally for the ${ }^{12} \mathrm{C}\left(v_{\mu}, \mu^{-}\right){ }^{12} \mathrm{~N}^{*}$ with the muon neutrinos originating in the pion decay-in-flight at LAMPF we have $\langle|\vec{q}|\rangle \simeq 200 \mathrm{MeV}$ and the typical excitation energy is $\omega \simeq 40 \mathrm{MeV}$. The excitation energies should be compared with the nuclear shell spacing $\hbar \omega \simeq 41 / A^{1 / 3} \mathrm{MeV}$, which for ${ }^{12} \mathrm{C}$ is equal to about $18 \mathrm{MeV}$. Thus, in order to describe all the above inclusive processes in the framework of the nuclear shell model, one would have to include fully and consistently at least all $2 \hbar \omega$ excitations, and possibly even the $3 \hbar \omega$ ones. This is not impossible, but represents a formidable task. On the other hand, the CRPA can easily handle such configuration spaces. Moreover, it properly describes the continuum nature of the final nucleus. Finally, as argued above, the crudeness with which the correlations of the $p$ shell nucleons are treated in the CRPA is expected to be relatively unimportant.

For the inclusive reaction ${ }^{12} \mathrm{C}\left(v_{e}, \mathrm{e}^{-}\right){ }^{12} \mathrm{~N}^{*}$, with $v_{e}$ neutrinos from the muon decay-at-rest the calculation gives [19] the cross section of $6.3 \times 10^{-42} \mathrm{~cm}^{2}$ using the Bonn potential based Gmatrix as the residual interaction, and $5.9 \times 10^{-42} \mathrm{~cm}^{2}$ with the schematic Migdal force. (The two different residual interactions are used so that one can estimate the uncertainty associated with this aspect of the problem.) Both are clearly compatible with the measured values of $6.4 \pm 1.45$ [stat] \pm 1.4 [syst] $\times 10^{-42} \mathrm{~cm}^{2}$ by the Karmen collaboration [16] (the more recent result gives somewhat smaller value $5.1 \pm 0.6 \pm 0.5$ [49]) and with $5.7 \pm 0.6[$ stat] \pm 0.6 [syst] $\times 10^{-42} \mathrm{~cm}^{2}$ obtained by the LSND collaboration [17] . If one wants to disregard the error bars (naturally, one should not do that), one can average the two calculated values 
as well as the two most recent measurements and perhaps conclude that the CRPA calculation seems to exceed the measured values by about 10-15\%. A similar tendency can be found, again with some degree of imagination, in the comparison of the muon capture rates discussed earlier.

So far we have found that the CRPA describes the inclusive reactions quite well. Other theoretical calculations, e.g. [23, 25] describe these reactions with equal success. This is no longer the case when we consider the reaction ${ }^{12} \mathrm{C}\left(v_{\mu}, \mu\right){ }^{12} \mathrm{~N}^{*}$ with the higher energy $v_{\mu}$ neutrinos from the pion decay-in-flight. This reaction involves larger momentum transfers and populates states higher up in the continuum of ${ }^{12} \mathrm{~N}$. The CRPA calculation [19, 20] gives the cross section of $19.2 \times 10^{-40} \mathrm{~cm}^{2}$, considerably larger than the measured [17] value of $11.3 \pm 0.3$ [stat] \pm 1.8 [syst] in the same units. The origin of the discrepancy is not clear, but as stressed in the discussion of the exclusive reaction, the $v_{\mu}$ flux normalization is not a likely culprit. While reference [22] confirms our result, reference [24] gets a value close to the experiment by using a generalization of the EPT approach.

Other recent theoretical calculations span the region between the CRPA and experiment. So, Singh et al [23] give $16.65 \pm 1.37 \times 10^{-40} \mathrm{~cm}^{2}$, clearly higher than the experiment but somewhat lower than the CRPA. On the other hand, reference [25] gives 13.5-15.2 in the same units, a value which is even closer to the experiment. The main difference in that work is the inclusion of pairing which is not expected to represent a substantial effect.

This discrepancy has been with us for quite some time now. It clearly exceeds the 10-15\% perhaps suggested by the lower energy inclusive reactions discussed above. It would be very important to perform a large scale shell model calculation, including up to $3 \hbar \omega$ excitations, to put the matter to rest. Attempts to do that are in [30, 31].

\section{Supernova neutrinos}

One of the most important applications of the neutrino-nucleus interaction is the detection of supernova neutrinos. In this section, after a few introductory remarks, we describe several examples of the calculated charged and neutral current cross sections on oxygen, argon, iron and lead. All these nuclei are being considered (or actually are already used) as targets for the supernova neutrino detection. Some of these cross sections are amenable to tests using the spallation neutron source, since the neutrino spectra of stopped pions and muons are quite similar to the expected neutrino spectra from the core collapse supernovae. The general review of the field can be found in [32].

Supernova neutrinos from SN1987a, presumably all $\bar{v}_{e}$, had been observed by the Kamiokande and IMB detectors [33, 34] and have confirmed the general supernova picture. However, the supernova models predict distinct differences in the neutrino distributions for the various flavours and thus a more restrictive test of the current supernova theory requires the abilities of neutrino spectroscopy by the neutrino detectors. Current (e.g. Superkamiokande, SNO, KamLAND) and future detectors (including the proposed OMNIS [35] and LAND [36] projects) have this capability and will be able to distinguish between the different neutrino flavours and determine their individual spectra. For the water Čerenkov detectors (SNO and Superkamiokande) $v_{x}$ neutrinos can be detected by specific neutral-current events [13, 37], while the OMNIS and LAND detectors are proposed to detect neutrons spalled from target nuclei by charged- and neutral-current neutrino interactions.

Theoretical models predict characteristic differences in the neutrino distributions for the various neutrino flavours (so-called temperature hierarchy). The $\mu$ and $\tau$ neutrinos and their antiparticles (combinely referred to as $v_{x}$ ) decouple deepest in the star, i.e. at the highest temperature, and have an average energy of $\bar{E}_{v} \simeq 25 \mathrm{MeV}$. The $v_{e}$ and $\bar{v}_{e}$ neutrinos interact 
with the neutron-rich matter via $v_{e}+n \rightarrow p+\mathrm{e}^{-}$and $\bar{v}_{e}+p \rightarrow n+\mathrm{e}^{+}$; the $\bar{v}_{e}$ neutrinos have a higher average energy $\left(\bar{E}_{v} \simeq 16 \mathrm{MeV}\right)$ than the $v_{e}$ neutrinos $\left(\bar{E}_{v} \simeq 11 \mathrm{MeV}\right)$. Clearly an observational verification of this temperature hierarchy would establish a strong test of the supernova models. The distribution of the various supernova neutrino species is usually described by the pinched Fermi-Dirac spectrum

$$
n\left(E_{v}\right)=\frac{1}{F_{2}(\alpha) T^{3}} \frac{E_{v}^{2}}{\exp \left[\left(E_{v} / T\right)-\alpha\right]+1},
$$

where $T, \alpha$ are parameters fitted to numerical spectra, and $F_{2}(\alpha)$ normalizes the spectrum to unit flux. The transport calculations of Janka [39] yield spectra with $\alpha \sim 3$ for all neutrino species. While this choice also gives good fits to the $v_{e}$ and $\bar{v}_{e}$ spectra calculated by Wilson and Mayle [40], their $v_{x}$ spectra favour $\alpha=0$. In the following we will present results for charged- and neutral current reactions on several target nuclei for both values of $\alpha$. In particular we will include results for those $(T, \alpha)$ values which are currently favoured for the various neutrino types ( $\mathrm{T}$ in $\mathrm{MeV}):(T, \alpha)=(4,0)$ and $(3,3)$ for $v_{e}$ neutrinos, $(5,0)$ and $(4,3)$ for $\bar{v}_{e}$ neutrinos and $(8,0)$ and $(6.26,3)$ for $v_{x}$ neutrinos. However, it is worthwhile pointing out that the degree of separation in energy of the different flavours is somewhat model dependent, as shown, e.g. in [38]. It is therefore even more important to determine the relevant parameters experimentally.

As stated above, it is usually sufficient to evaluate the various neutrino-induced reaction cross sections within the RPA. However, the RPA often does not recover sufficient nucleonnucleon correlations to reliably reproduce the quenching and fragmentation of the GamowTeller (GT) strength distribution in nuclei. Therefore, the response of the $\lambda^{\pi}=1^{+}$operator should be evaluated on the basis of an interacting shell model, if such calculations are feasible. While the double-magic nucleus ${ }^{16} \mathrm{O}$ does not allow GT excitations, shell model calculations in reliably large model spaces are possible for ${ }^{40} \mathrm{Ar}$ and ${ }^{56} \mathrm{Fe}$. For ${ }^{208} \mathrm{~Pb} \mathrm{GT}$ transitions are Pauli-blocked for $\left(\bar{v}_{e}, \mathrm{e}^{+}\right)$reactions. The modelling of GT transitions in the $\left(v_{e}, \mathrm{e}^{-}\right)$reactions on ${ }^{208} \mathrm{~Pb}$ would require much too large model spaces; these transitions must also be evaluated within the RPA approach.

In the following we will refer to a 'hybrid model' if the allowed transitions have been studied based on the interacting shell model, while the forbidden transitions were calculated within the random phase approximation. The studies for ${ }^{40} \mathrm{Ar}$ and ${ }^{56} \mathrm{Fe}$ are performed in such a hybrid model, while the ones for ${ }^{16} \mathrm{O}$ and ${ }^{208} \mathrm{~Pb}$ are RPA calculations for all multipoles.

We note that the GT operator corresponds to the $\lambda^{\pi}=1^{+}$operator only in the limit of momentum transfer $q \rightarrow 0$. As has been pointed out in [41, 42], the consideration of the finitemomentum transfer in the operator results in a reduction of the cross sections. To account for the effect of the finite momentum transfer we performed RPA calculations for the $\lambda^{\pi}=1^{+}$ multipole operator at finite momentum transfer $q$ (i.e. $\lambda(q))$ and for $q=0$ (i.e., $\lambda(q=0)$ ) and scaled the shell model GT strength distribution by the ratio of $\lambda(q)$ and $\lambda(q=0)$ RPA cross sections. The correction is rather small for $v_{e}$ neutrinos stemming from muon-decay-at-rest neutrinos (e.g., for LSND and Karmen) or for supernova $v_{e}$ neutrinos. The correction is, however, sizeable for higher neutrino energies.

\subsection{Oxygen}

Observation of neutrinos from the SN1987A did not allow us to test in detail the neutrino distribution and, in particular, it gave no information about $v_{x}$ neutrinos which, as we discussed above, should decouple deepest in the star. The observability of supernova neutrinos has significantly improved since the Superkamiokande (SK) detector, with a threshold of $5 \mathrm{MeV}$ and with 30 times the size of Kamiokande, became operational [43]. 


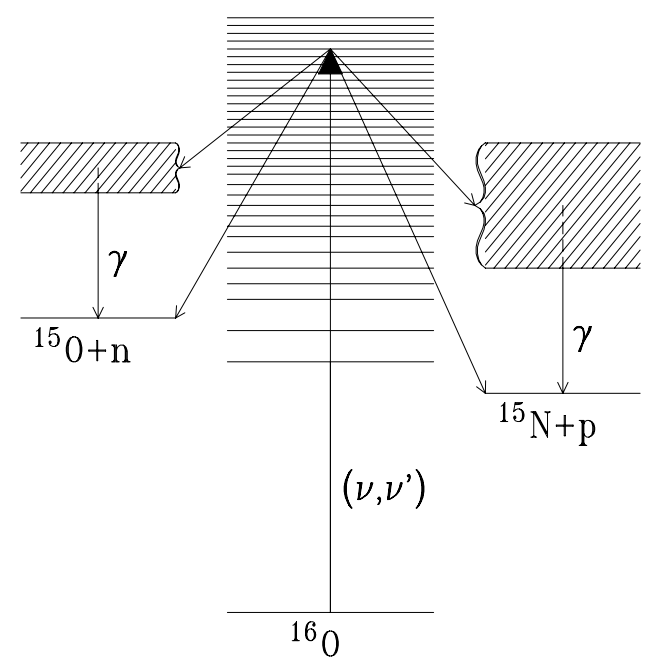

Figure 6. Schematic illustration of the detection scheme for the neutral current detection in water Čerenkov detectors.

Clearly, many of the primary neutral- and charged-current $v$-induced reactions in SK occur on ${ }^{16} \mathrm{O}$. Atmospheric neutrinos and also supernova $v_{x}$ neutrinos have high enough energies so that the final nucleus in the primary reaction will be in an excited state which will then decay by a cascade of particle and $\gamma$ emissions. This fact has been used to propose a signal for the observation of $v_{x}$ neutrinos in water Čerenkov detectors [13]. Schematically the detection scheme works as follows (see figure 6). Supernova $v_{x}$ neutrinos, with average energies of $\approx 25 \mathrm{MeV}$, will predominantly excite $1^{-}$and $2^{-}$giant resonances in ${ }^{16} \mathrm{O}$ via the ${ }^{16} \mathrm{O}\left(v_{x}, v_{x}^{\prime}\right){ }^{16} \mathrm{O}^{\star}$ neutral current reaction [10]. These resonances are above the particle thresholds and will mainly decay by proton and neutron emission. Although these decays will be dominantly to the ground states of ${ }^{15} \mathrm{~N}$ and ${ }^{15} \mathrm{O}$, respectively, some of them will go to excited states in these nuclei. In turn, if these excited states are below the particle thresholds in ${ }^{15} \mathrm{~N}\left(E^{\star}<10.2 \mathrm{MeV}\right)$ or ${ }^{15} \mathrm{O}\left(E^{\star}<7.3 \mathrm{MeV}\right)$, they will decay by $\gamma$ emission. As the first excited states in both of these mirror nuclei $\left(E^{\star}=5.27 \mathrm{MeV}\right.$ in ${ }^{15} \mathrm{~N}$ and $E^{\star}=5.18 \mathrm{MeV}$ in ${ }^{15} \mathrm{O}$ ) are at energies larger than the SK detection threshold, all of the bound excited states in ${ }^{15} \mathrm{~N}$ and ${ }^{15} \mathrm{O}$ below will emit photons which can be observed in SK.

Based on a calculation which combines the continuum RPA with the statistical model [13], Superkamiokande is expected to observe about $700 \gamma$ events in the energy window $E=5-10 \mathrm{MeV}$, induced by $v_{x}$ neutrinos (with a FD distribution of $T=8 \mathrm{MeV}$ ), for a supernova going off at $10 \mathrm{kpc}\left(\approx 3 \times 10^{4}\right.$ light years or the distance to the galactic centre) (see figure 7). This is to be compared with a smooth background of about 270 positron events from the $\bar{v}_{e}+p \rightarrow n+\mathrm{e}^{+}$reaction in the same energy window. The number of events produced by supernova $v_{x}$ neutrinos via the scheme proposed here is larger than the total number of events expected from $v_{x}$-electron scattering (about 80 events [43]). More importantly, the $\gamma$ signal can be unambiguously identified from the observed spectrum in the SK detector, in contrast to the more difficult identification from $v_{x}$-electron scattering.

The cascades of decays, following the inelastic excitation of ${ }^{16} \mathrm{O}$ by atmospheric or supernova $v_{x}$ neutrinos, can also result in the production of $\beta$-unstable nuclei. If the $Q_{\beta}$ values of these nuclei are above the observational threshold energy in SK ( $~ 5 \mathrm{MeV})$, these decays might be detectable, and since they are usually delayed, might offer an additional 


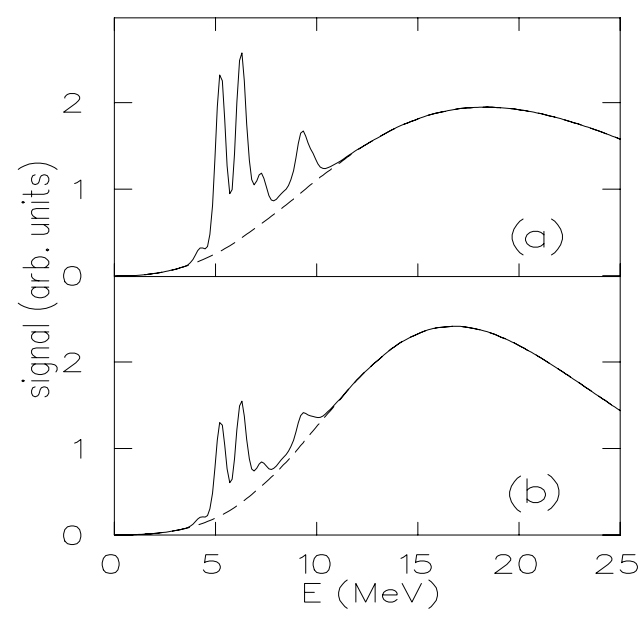

Figure 7. Signal expected from supernova neutrinos in a water Čerenkov detector calculated for two different types of neutrino distributions (without chemical potential (above) and with chemical potential $\alpha=3 T$ and temperatures $T=6.26 \mathrm{MeV}$ (for $v_{x}$ ) and $T=4 \mathrm{MeV}$ (for $\bar{\nu}_{e}$ )). The bulk of the signal stems from $\bar{v}_{e}$ neutrinos reacting with protons, while the $v_{x}$ neutrinos induce the superimposed signal at energies $E=5-10 \mathrm{MeV}$ (from [13]).

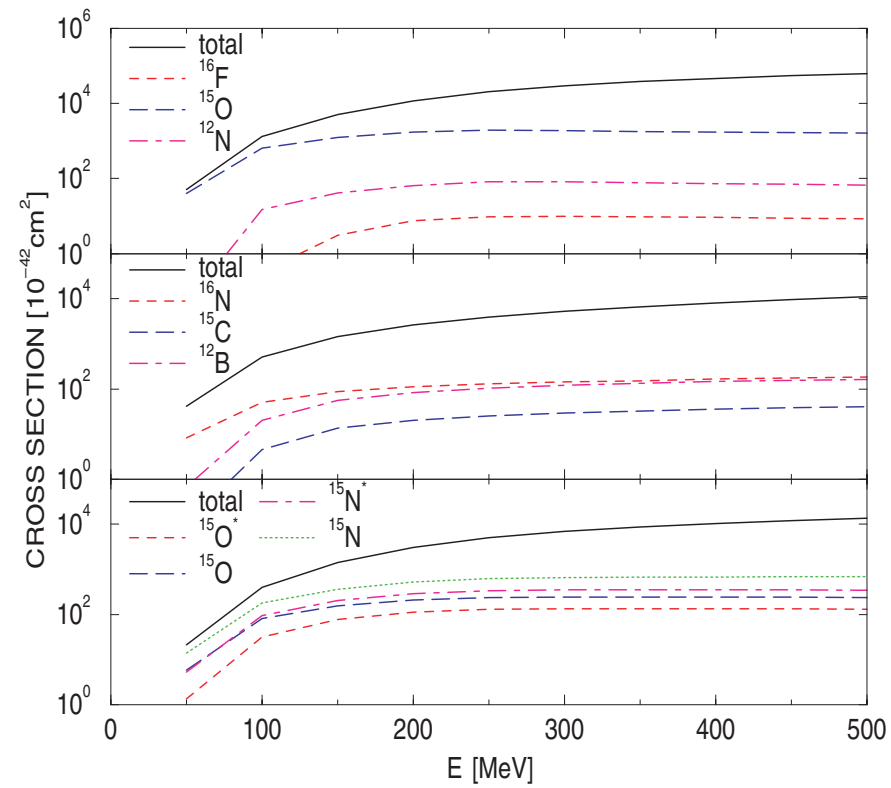

Figure 8. Total and partial cross sections to selected $\beta$-unstable nuclear ground states for $\left(v_{e}, \mathrm{e}^{-}\right)$ (upper), $\left(\bar{v}_{e}, \mathrm{e}^{+}\right)$(middle) and $\left(v, v^{\prime}\right)$ (lower part) reactions on ${ }^{16} \mathrm{O}$ as function of neutrino energy. As explained in the text, the symbols ${ }^{15} \mathrm{O}^{*}$ and ${ }^{15} \mathrm{~N}^{*}$ in the lower panel denote the partial cross sections leading through particle-bound excited states in these nuclei.

characteristic signature of the neutrino-induced reactions. As possible candidates ${ }^{16} \mathrm{~N},{ }^{15} \mathrm{C}$, ${ }^{12} \mathrm{~B}$ and ${ }^{12} \mathrm{~N}$ have been identified $[44,45]$.

Figure 8 shows the partial $\left(\bar{v}_{e}, \mathrm{e}^{+}\right)$and $\left(v_{e}, \mathrm{e}^{-}\right)$cross sections leading to the $\beta$-unstable ${ }^{16} \mathrm{~N},{ }^{15} \mathrm{C}$ and ${ }^{12} \mathrm{~B}$ and ${ }^{16} \mathrm{~F},{ }^{15} \mathrm{O}$ and ${ }^{12} \mathrm{~N}$ ground states in the final channel; these cross sections reflect the sum over all cross sections with particle-bound states of these nuclei as these excited 
Table 2. Partial cross sections for neutral-current neutrino-induced reactions on ${ }^{16} \mathrm{O}$. A FermiDirac distribution with $T=8 \mathrm{MeV}$ and zero chemical potential, which is typical for supernova $v_{\mu}$ and $v_{\tau}$ neutrinos and their antiparticles, has been assumed. The cross section, in units of $10^{-42} \mathrm{~cm}^{2}$, represents the average for neutrino and antineutrino reactions, and the exponents are given in parentheses. The asterisks indicate that the cross sections have been summed over all particle-bound states.

\begin{tabular}{ll}
\hline Neutrino reaction & Partial $\sigma$ \\
\hline Total & $5.19(00)$ \\
${ }^{16} \mathrm{O}\left(v, v^{\prime} \gamma\right){ }^{16} \mathrm{O}^{*}$ & $3.19(-3)$ \\
${ }^{16} \mathrm{O}\left(v, v^{\prime} \mathrm{n}\right){ }^{15} \mathrm{O}(\mathrm{gs})$ & $9.73(-1)$ \\
${ }^{16} \mathrm{O}\left(v, v^{\prime} \mathrm{p}\right){ }^{15} \mathrm{~N}(\mathrm{gs})$ & $1.85(00)$ \\
${ }^{16} \mathrm{O}\left(v, v^{\prime} n \gamma\right){ }^{15} \mathrm{O}^{*}$ & $3.48(-1)$ \\
${ }^{16} \mathrm{O}\left(v, v^{\prime} \mathrm{nn}\right){ }^{14} \mathrm{O}^{*}$ & $6.11(-3)$ \\
${ }^{16} \mathrm{O}\left(v, v^{\prime} \mathrm{np}\right){ }^{14} \mathrm{~N}^{*}$ & $4.40(-1)$ \\
${ }^{16} \mathrm{O}\left(v, v^{\prime} p \gamma\right){ }^{15} \mathrm{~N}^{*}$ & $1.29(00)$ \\
${ }^{16} \mathrm{O}\left(v, v^{\prime} \mathrm{pp}\right){ }^{14} \mathrm{C}^{*}$ & $8.35(-2)$ \\
$\left.{ }^{16} \mathrm{O}\left(v, v^{\prime} p \alpha\right)\right)^{11} \mathrm{~B}^{*}$ & $9.15(-2)$ \\
${ }^{16} \mathrm{O}\left(v, v^{\prime} n \alpha\right){ }^{11} \mathrm{C}^{*}$ & $3.88(-2)$ \\
\hline
\end{tabular}

Table 3. Partial cross sections for charged-current neutrino-induced reactions on ${ }^{16} \mathrm{O}$. Fermi-Dirac distributions with $T=4 \mathrm{MeV}$ and $T=8 \mathrm{MeV}$ and zero chemical potential have been assumed. The first is typical for supernova $v_{e}$ neutrinos, while the second can occur for complete $v_{e} \leftrightarrow v_{\mu}$ oscillations. The cross sections are given in units of $10^{-42} \mathrm{~cm}^{2}$, exponents are given in parentheses.

\begin{tabular}{lll}
\hline Neutrino reaction & $\sigma, T=4 \mathrm{MeV}$ & $\sigma, T=8 \mathrm{MeV}$ \\
\hline Total & $1.91(-1)$ & $1.37(+1)$ \\
${ }^{16} \mathrm{O}\left(v, \mathrm{e}^{-} \mathrm{p} \gamma\right){ }^{15} \mathrm{O}(\mathrm{gs})$ & $1.21(-1)$ & $6.37(00)$ \\
${ }^{16} \mathrm{O}\left(v, \mathrm{e}^{-} \mathrm{p} \gamma\right)^{15} \mathrm{O}^{*}$ & $4.07(-2)$ & $3.19(00)$ \\
${ }^{16} \mathrm{O}\left(v, \mathrm{e}^{-} \mathrm{np}\right){ }^{14} \mathrm{O}^{*}$ & $3.92(-4)$ & $1.76(-1)$ \\
${ }^{16} \mathrm{O}\left(v, \mathrm{e}^{-} \mathrm{pp}\right){ }^{14} \mathrm{~N}^{*}$ & $2.61(-2)$ & $3.26(00)$ \\
${ }^{16} \mathrm{O}\left(v, \mathrm{e}^{-} \alpha\right){ }^{12} \mathrm{~N}^{*}$ & $1.16(-3)$ & $1.31(-1)$ \\
${ }^{16} \mathrm{O}\left(v, \mathrm{e}^{-} p \alpha\right){ }^{11} \mathrm{C}^{*}$ & $1.55(-3)$ & $5.66(-1)$ \\
${ }^{16} \mathrm{O}\left(v, \mathrm{e}^{-} p n \alpha\right){ }^{10} \mathrm{C}^{*}$ & $1.11(-6)$ & $3.28(-3)$ \\
\hline
\end{tabular}

states will fast decay to the ground state by $\gamma$ emission. Our calculations have been performed up to neutrino energies $E_{v}=500 \mathrm{MeV}$. At higher energies the total cross sections can be obtained from a relativistic Fermi gas model [46], including, however, additional channels like pion production.

The results make it possible to draw some interesting conclusions. While the total cross sections increase with neutrino energies, most of this increase goes into new channels which open up with increasing neutrino energy and the partial cross sections leading to definite states have the tendency to saturate. Thus our partial cross sections to these states, obtained for, say, $E_{v}=500 \mathrm{MeV}$, can be used to derive upper limits for the corresponding branching ratios expected for atmospheric neutrinos which, on average, have even larger energies. The total and selected partial cross sections for the neutral- and charged-current reactions on ${ }^{16} \mathrm{O}$ for different supernova neutrino spectra are summarized in tables 2-4.

A detailed discussion of the various cross sections is given in [45]. In general, these RPA cross sections are significantly smaller than estimated in [44]. In particular, they indicate that, for typical atmospheric neutrino energies, the partial cross sections leading to $\beta$-unstable nuclei are, unfortunately, too small so that the observation of the decay of these nuclei does not constitute an additional viable signal for neutrino-induced reactions in Superkamiokande. 
Table 4. Partial cross sections for charged-current antineutrino-induced reactions on ${ }^{16} \mathrm{O}$. FermiDirac distributions with $T=5 \mathrm{MeV}$ and $T=8 \mathrm{MeV}$ and zero chemical potential have been assumed. The first is typical for supernova $\bar{v}_{e}$ neutrinos, while the second can occur for complete $\bar{v}_{e} \leftrightarrow \bar{v}_{\mu}$ oscillations. The cross sections are given in units of $10^{-42} \mathrm{~cm}^{2}$, exponents are given in parentheses.

\begin{tabular}{lll}
\hline Neutrino reaction & $\sigma, T=5 \mathrm{MeV}$ & $\sigma, T=8 \mathrm{MeV}$ \\
\hline Total & $1.05(00)$ & $9.63(00)$ \\
${ }^{16} \mathrm{O}\left(\bar{v}, \mathrm{e}^{+}\right){ }^{16} \mathrm{~N}(\mathrm{gs})$ & $3.47(-1)$ & $2.15(00)$ \\
${ }^{16} \mathrm{O}\left(\bar{v}, \mathrm{e}^{+} \mathrm{n}\right){ }^{15} \mathrm{~N}(\mathrm{gs})$ & $5.24(-1)$ & $4.81(00)$ \\
${ }^{16} \mathrm{O}\left(\bar{v}, \mathrm{e}^{+} \mathrm{n} \gamma\right)^{15} \mathrm{~N}^{*}$ & $1.47(-1)$ & $1.90(00)$ \\
${ }^{16} \mathrm{O}\left(\bar{v}, \mathrm{e}^{+} \mathrm{np}\right){ }^{14} \mathrm{C}^{*}$ & $4.56(-3)$ & $1.38(-1)$ \\
${ }^{16} \mathrm{O}\left(\bar{v}, \mathrm{e}^{+} \mathrm{nn}\right){ }^{14} \mathrm{~N}^{*}$ & $5.50(-3)$ & $1.81(-1)$ \\
${ }^{16} \mathrm{O}\left(\bar{v}, \mathrm{e}^{+} \alpha\right){ }^{12} \mathrm{~B}^{*}$ & $1.07(-2)$ & $1.91(-1)$ \\
${ }^{16} \mathrm{O}\left(\bar{v}, \mathrm{e}^{+} n \alpha\right){ }^{11} \mathrm{~B}^{*}$ & $6.20(-3)$ & $2.16(-1)$ \\
\hline
\end{tabular}

For supernova $\bar{v}_{e}$ neutrinos, however, the ${ }^{16} \mathrm{O}\left(\bar{v}_{e}, \mathrm{e}^{+}\right){ }^{16} \mathrm{~N}$ reaction can produce an observable additional signal in Superkamiokande for supernovae from within our galaxy. The reaction leads to excited states in ${ }^{16} \mathrm{~N}$ at rather low energies, which then dominantly decay by neutron emission. However, as can be seen in table 4 , a sizable fraction of the $\left(\bar{v}_{e}, \mathrm{e}^{+}\right)$ reactions also excite particle-bound states in ${ }^{16} \mathrm{~N}$, followed then by the $\beta$ decay of the ${ }^{16} \mathrm{~N}$ ground state. Assuming the standard antineutrino supernova spectrum (with $T=5 \mathrm{MeV}$ ) the partial cross section of $3.5 \times 10^{-43} \mathrm{~cm}^{2}$ corresponds to about 40 supernova $\bar{v}_{e}$-induced events in SK leading to the ${ }^{16} \mathrm{~N}$ ground state and which can be identified by the delayed $\beta$ decay for a hypothetical supernova in the galactic centre. Note, however, that this event rate corresponds to less than $1 \%$ of the total supernova neutrino rate in SK, with positrons being produced by the $\bar{\nu}_{e}+p \rightarrow \mathrm{e}^{+}+n$ reaction giving the dominating signal. Thus, it is unlikely that $\beta$ decays from ${ }^{16} \mathrm{~N}$, generated by ${ }^{16} \mathrm{O}\left(\bar{v}_{e}, \mathrm{e}^{+}\right){ }^{16} \mathrm{~N}$ in the supernova $1987 \mathrm{~A}$, were observed by the Kamiokande detector. The partial ${ }^{16} \mathrm{O}\left(\bar{v}_{e}, \mathrm{e}^{+}\right){ }^{16} \mathrm{~N}$ reaction cross section increases by more than a factor of 6 , if complete $\bar{v}_{e} \leftrightarrow \bar{v}_{\mu, \tau}$ oscillations occur, constituting then a very sizable and clean signal for SK.

Another interesting reaction, leading to an observable $\beta$ decay in $\mathrm{SK}$, is ${ }^{16} \mathrm{O}\left(\overline{\mathrm{v}}_{e}, \mathrm{e}^{+} \alpha\right){ }^{12} \mathrm{~B}$. For the standard spectrum we find a partial cross section of $1.1 \times 10^{-44} \mathrm{~cm}^{2}$, which increases to $1.9 \times 10^{-43} \mathrm{~cm}^{2}$ for the case of oscillations. This translates into $O(10)^{12} \mathrm{~B}$ decays in the SK detector for a supernova in the galactic center at $10 \mathrm{kpc}$ and if complete $\bar{v}_{e} \leftrightarrow \bar{v}_{\mu, \tau}$ oscillations occur.

\subsection{Argon}

The proposed ICARUS detector uses a liquid Argon technique providing energy and direction measurements for electrons, muons, pions and protons [47]. Observation of supernova neutrinos is also anticipated in the detector.

The detection and analysis of supernova neutrinos by the ICARUS detector requires knowledge of the neutrino-induced cross sections on ${ }^{40} \mathrm{Ar}$ for neutrinos and antineutrinos with energies up to about $100 \mathrm{MeV}$. At low neutrino energies the $\left(v_{e}, \mathrm{e}^{-}\right)$reaction is dominated by allowed GT transitions. However, the calculation of this cross section constitutes a quite challenging nuclear structure problem. In the independent particle model (IPM), ${ }^{40} \mathrm{Ar}$ $(Z=18, N=22$ ) corresponds to a two-hole proton configuration in the $s d$ shell and a two-particle neutron configuration in the $p f$ shell. One thus has to expect that cross-shell correlations will strongly influence the structure of the low-lying states in ${ }^{40} \mathrm{Ar}$, including the 


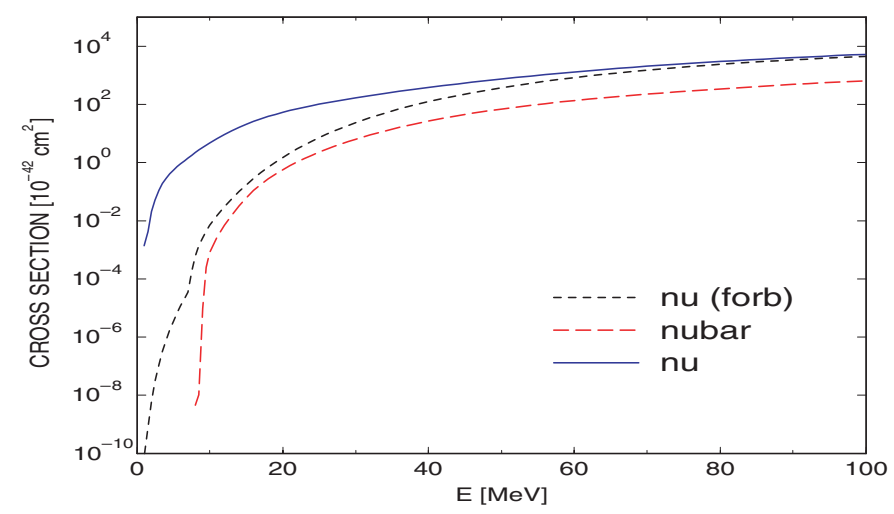

Figure 9. Total $\left(v_{e}, \mathrm{e}^{-}\right)$(solid) and $\left(\bar{v}_{e}, \mathrm{e}^{+}\right)$(dashed) cross sections on ${ }^{40} \mathrm{Ar}$, calculated within the RPA approach. The short-dashed line shows the forbidden contributions to the $\left(v_{e}, \mathrm{e}^{-}\right) \operatorname{cross}$ sections.

GT_ response of the ground state. To describe such correlations requires shell model studies in the complete $s d-p f$ shell which are currently not feasible by diagonalization methods. One therefore has to rely on truncated shell model calculations in which only a restricted number of particles are allowed to be excited from the $s d$ shell to the $p f$ shell. The best study to date has been performed by Ormand [48] who calculated the low-lying GT response of the ${ }^{40} \mathrm{Ar}$ ground state within a $2 \hbar \omega$ shell model diagonalization in which he considered 2-particle excitations from the $s d$ into the $p f$ shell, carefully avoiding spurious centre-of-mass excitations. His calculation is appropriate for solar neutrinos with energies $E_{v} \leqslant 14 \mathrm{MeV}$. Importantly, the shell model study showed that the GT transitions dominate over the allowed Fermi transition to the IAS in ${ }^{40} \mathrm{~K}$ at $4.38 \mathrm{MeV}$. However, due to the (rather severe) truncation of the model space this shell model calculation violates the Ikeda sum rule and hence misses GT strength at higher excitation energies in ${ }^{40} \mathrm{~K}$. This strength will be important for neutrinos (like those from a supernova) which have larger energies than solar neutrinos and can excite the daughter nucleus at higher energies. An appropriate shell model calculation which describes this GT strength at higher energies is currently not available. However, RPA studies of the ${ }^{40} \operatorname{Ar}\left(v_{e}, \mathrm{e}^{-}\right)^{40} \mathrm{~K}$ reaction have been performed considering allowed and forbidden multipoles up to $J=4$. The respective cross sections are shown in figure 9. It is evident that GT transitions dominate the $\left(v_{e}, \mathrm{e}^{-}\right)$cross sections for neutrino energies $E_{v}<50 \mathrm{MeV}$; at higher energies forbidden (in particular spin-dipole) transitions cannot be neglected.

In the IPM, the $\mathrm{GT}_{+}$strength for ${ }^{40} \mathrm{Ar}$ vanishes as all GT transitions, in which a proton is changed into a neutron, are Pauli-blocked. Although cross-shell correlations might introduce a non-vanishing $\mathrm{GT}_{+}$strength, it should be small. Hence it is reasonable to calculate the $\left(\bar{v}_{e}, \mathrm{e}^{+}\right)$cross section on ${ }^{40} \mathrm{Ar}$ within the RPA approach. The obtained results are also shown in figure 9.

\subsection{Iron}

The KARMEN collaboration used its sensitivity to the ${ }^{56} \mathrm{Fe}\left(v_{e}, \mathrm{e}^{-}\right){ }^{56} \mathrm{Co}$ background events to determine the cross section for this reaction for the DAR neutrino spectrum and obtained $\sigma=(2.56 \pm 1.08 \pm 0.43) \times 10^{-40} \mathrm{~cm}^{2}$ [49]. We calculate a result in close agreement, $\sigma=2.4 \times 10^{-40} \mathrm{~cm}^{2}[41]$.

We extended this investigation to the study of the charged- and neutral current reactions on ${ }^{56} \mathrm{Fe}$. To allow also for the exploration of potential oscillation scenarios we also evaluated 
Table 5. Total cross sections for neutral current neutrino scattering on ${ }^{56} \mathrm{Fe}$ for different neutrino energy spectra represented as Fermi-Dirac distributions. The cross sections are in units of $10^{-42} \mathrm{~cm}^{2}$ and are averaged over neutrinos and antineutrinos.

\begin{tabular}{llllllll}
\hline$(T, \alpha)$ & $(4,0)$ & $(6,0)$ & $(8,0)$ & $(10,0)$ & $(3,-3)$ & $(4,-3)$ & $(6.26,-3)$ \\
\hline${ }^{56} \mathrm{Fe}\left(v, v^{\prime} \gamma\right){ }^{56} \mathrm{Fe}$ & $2.5(0)$ & $9.8(0)$ & $1.7(1)$ & $2.8(1)$ & $1.2(0)$ & $4.4(0)$ & $1.6(1)$ \\
${ }^{56} \mathrm{Fe}\left(v, v^{\prime} \mathrm{n}\right){ }^{55} \mathrm{Fe}$ & $8.9(-1)$ & $6.7(0)$ & $2.2(1)$ & $5.0(1)$ & $2.8(-1)$ & $1.7(0)$ & $1.4(1)$ \\
${ }^{56} \mathrm{Fe}\left(v, v^{\prime} \mathrm{p}\right){ }^{55} \mathrm{Mn}$ & $1.2(-1)$ & $1.0(0)$ & $3.6(0)$ & $9.3(0)$ & $3.4(-2)$ & $2.3(-1)$ & $2.2(0)$ \\
${ }^{56} \mathrm{Fe}\left(v, v^{\prime} \alpha\right){ }^{52} \mathrm{Cr}$ & $2.4(-2)$ & $1.9(-1)$ & $6.6(-1)$ & $1.7(0)$ & $6.4(-3)$ & $4.4(-2)$ & $4.0(-1)$ \\
${ }^{56} \mathrm{Fe}\left(v, v^{\prime}\right) \mathrm{X}$ & $3.6(0)$ & $1.8(1)$ & $4.3(1)$ & $8.9(1)$ & $1.5(0)$ & $6.3(0)$ & $3.3(1)$ \\
\hline
\end{tabular}

Table 6. Total cross sections for charged current neutrino scattering on ${ }^{56} \mathrm{Fe}$ for different neutrino energy spectra represented as Fermi-Dirac distributions. The cross sections are in units of $10^{-42} \mathrm{~cm}^{2}$.

\begin{tabular}{llllllll}
\hline$(T, \alpha)$ & $(4,0)$ & $(6,0)$ & $(8,0)$ & $(10,0)$ & $(3,-3)$ & $(4,-3)$ & $(6.26,-3)$ \\
\hline${ }^{56} \mathrm{Fe}\left(v_{e}, \mathrm{e}^{-} \gamma\right)^{56} \mathrm{Co}$ & $9.8(0)$ & $3.1(1)$ & $6.1(1)$ & $1.3(2)$ & $7.7(0)$ & $2.1(1)$ & $7.5(1)$ \\
${ }^{56} \mathrm{Fe}\left(v_{e}, \mathrm{e}^{-} \mathrm{n}\right){ }^{55} \mathrm{Co}$ & $7.5(-1)$ & $8.0(0)$ & $3.2(1)$ & $8.1(1)$ & $2.5(-1)$ & $1.7(0)$ & $2.0(1)$ \\
${ }^{56} \mathrm{Fe}\left(v_{e}, \mathrm{e}^{-} \mathrm{p}\right)^{55} \mathrm{Fe}$ & $5.4(0)$ & $3.2(1)$ & $9.7(1)$ & $1.7(2)$ & $9.2(-1)$ & $5.1(0)$ & $4.7(1)$ \\
${ }^{56} \mathrm{Fe}\left(v_{e}, \mathrm{e}^{-} \alpha\right){ }^{52} \mathrm{Mn}$ & $6.1(-2)$ & $9.7(-1)$ & $4.8(0)$ & $1.5(1)$ & $3.0(-2)$ & $2.1(-1)$ & $2.9(0)$ \\
${ }^{56} \mathrm{Fe}\left(v_{e}, \mathrm{e}^{-}\right) \mathrm{X}$ & $1.6(1)$ & $7.2(1)$ & $1.9(2)$ & $4.0(2)$ & $8.9(0)$ & $2.8(1)$ & $1.4(2)$ \\
${ }^{56} \mathrm{Fe}\left(\bar{v}_{e}, \mathrm{e}^{+} \gamma\right){ }^{56} \mathrm{Mn}$ & $2.3(0)$ & $8.4(0)$ & $1.8(1)$ & $3.1(1)$ & $1.4(0)$ & $4.1(0)$ & $1.6(1)$ \\
${ }^{56} \mathrm{Fe}\left(\bar{v}_{e}, \mathrm{e}^{+} \mathrm{n}\right)^{55} \mathrm{Mn}$ & $4.2(-1)$ & $4.0(0)$ & $1.6(1)$ & $4.0(1)$ & $1.2(-1)$ & $7.9(-1)$ & $9.1(0)$ \\
${ }^{56} \mathrm{Fe}\left(\bar{v}_{e}, \mathrm{e}^{+} \mathrm{p}\right)^{55} \mathrm{Cr}$ & $4.5(-3)$ & $6.2(-2)$ & $3.2(-1)$ & $9.9(-1)$ & $9.3(-4)$ & $8.5(-3)$ & $1.5(-1)$ \\
${ }^{56} \mathrm{Fe}\left(\bar{v}_{e}, \mathrm{e}^{+} \alpha\right)^{52} \mathrm{~V}$ & $1.1(-3)$ & $1.7(-2)$ & $9.5(-2)$ & $3.1(-1)$ & $2.0(-4)$ & $2.1(-3)$ & $4.3(-2)$ \\
${ }^{56} \mathrm{Fe}\left(\bar{v}_{e}, \mathrm{e}^{+}\right) \mathrm{X}$ & $2.8(0)$ & $1.2(1)$ & $3.4(1)$ & $7.2(1)$ & $1.6(0)$ & $4.9(0)$ & $2.5(1)$ \\
\hline
\end{tabular}

the cross sections and the knockout neutron yields for various supernova neutrino spectra. Table 5 summarizes the total and partial cross sections for neutral current reactions on ${ }^{56} \mathrm{Fe}$. For ${ }^{56} \mathrm{Fe}$ the neutron and proton thresholds open at $11.2 \mathrm{MeV}$ and $10.18 \mathrm{MeV}$, respectively. But despite the slightly higher threshold energy, the additional Coulomb repulsion in the proton threshold makes the neutron channel the dominating decay mode. The total and partial cross sections for charged current $\left(v_{e}, \mathrm{e}^{-}\right)$and $\left(\bar{v}_{e}, \mathrm{e}^{+}\right)$reactions on ${ }^{56} \mathrm{Fe}$ are listed in table 6 . For the standard supernova $\nu_{e}$ spectrum $((T, \alpha)=(4,0))$, the low-energy excitation spectrum is relatively strongly weighted by phase space. Hence, in that case the $v_{e}$-induced reaction on ${ }^{56} \mathrm{Fe}$ leads dominantly to particle-bound states $(\sim 60 \%)$ and therefore decays by $\gamma$ emission.

The work of [41] has been extended to other iron isotopes, ${ }^{52-60} \mathrm{Fe}$, by Toivanen et al [50]. The respective total charged-current $\left(v, \mathrm{e}^{-}\right)$and neutral-current $\left(v, v^{\prime}\right)$ cross sections for typical supernova neutrino spectra (i.e. $T=4 \mathrm{MeV}$ for $v_{e}$ neutrinos and $T=8 \mathrm{MeV}$ for $v_{x}$ neutrinos) are shown in figures 10 and 11. Additionally these figures show the partial cross sections for the decay into the proton and neutron channels. Since for the isotopes ${ }^{52-56}$ Co the proton threshold is lower than the neutron threshold, the preferred decay mode for the $\left(v_{e}, \mathrm{e}^{-}\right)$ reaction on ${ }^{52-56} \mathrm{Fe}$ is by proton emission. This is reversed for ${ }^{58-60} \mathrm{Co}$, where decay into the neutron channel is preferred. Because parts of the Gamow-Teller and the Fermi strengths are located below the particle thresholds, these states decay by gamma emission which accounts basically for the difference between the total cross section and the sum of the partial proton and neutron decay cross sections. Decays into the $\alpha$ channel are unfavoured.

For the neutral-current reactions decay by proton emission is favoured in the proton-rich nuclei ${ }^{52-54} \mathrm{Fe}$. However, as the neutron threshold decreases with increasing mass number along the isotope chain, while the proton threshold energy increases, the probability for decay 


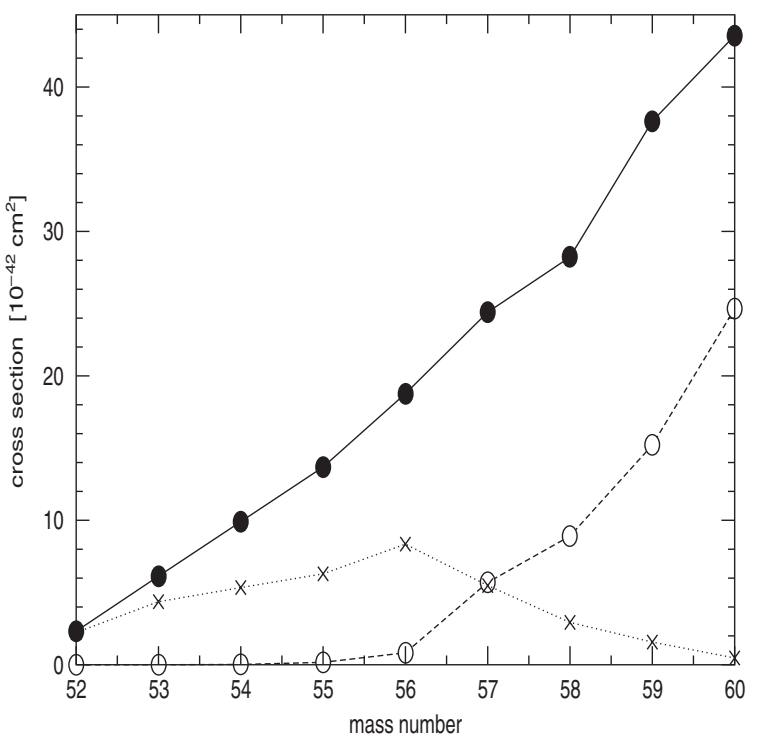

Figure 10. Total charged current cross section for $v_{e}$ on ${ }^{52-60} \mathrm{Fe}$ (filled circles connected by the full line) and the partial neutron (empty circles, dashed line) and proton (crosses, dotted line) spallation cross sections. The difference between the total and the two partial cross sections gives the gamma emission cross section since the $\alpha$ channel is negligible. The $v_{e}$ spectrum with $T=$ $4 \mathrm{MeV}$ and $\alpha=0$ was used.

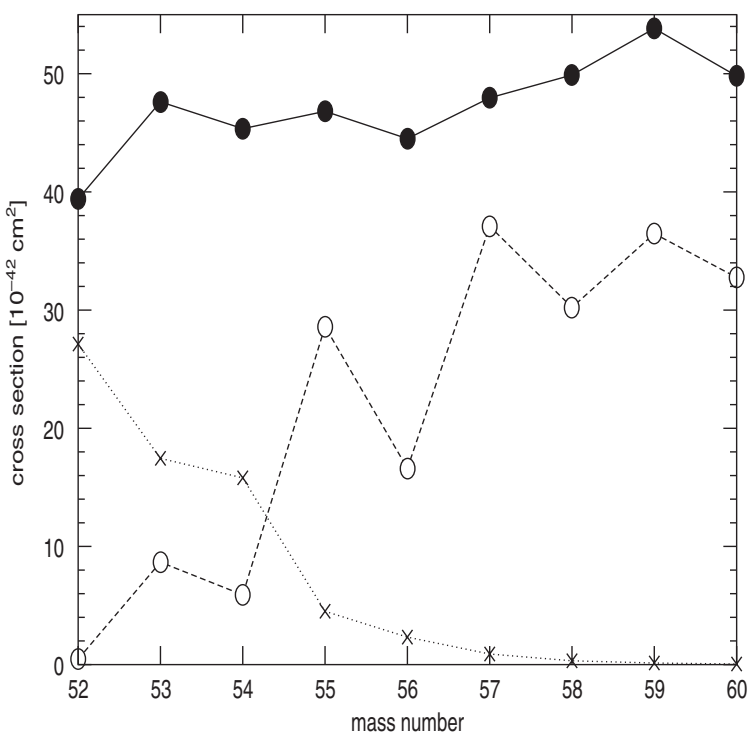

Figure 11. Total neutral current cross section for $v_{x}$ on ${ }^{52-60} \mathrm{Fe}$ averaged between $v$ and $\bar{v}$ (filled circles connected by the full line) and the partial neutron (empty circles, dashed line) and proton (crosses, dotted line) spallation cross sections. The difference between the total and the two partial cross sections gives the gamma emission cross section since the $\alpha$ channel is negligible. The $v_{x}$ spectra with $T=8 \mathrm{MeV}$ and $\alpha=0$ were used.

into the neutron channel increases at the expense of decay by proton emission. This is clearly reflected in the trend of the partial cross sections as function of neutron excess. Due to pairing 
Table 7. Total cross sections for charged-current neutrino scattering on ${ }^{208} \mathrm{~Pb}$ for different neutrino energy spectra represented as Fermi-Dirac distributions. The cross sections are given in units of $10^{-42} \mathrm{~cm}^{2}$.

\begin{tabular}{llllllll}
\hline$(T, \alpha)$ & $(4,0)$ & $(6,0)$ & $(8,0)$ & $(10,0)$ & $(3,3)$ & $(4,3)$ & $(6.26,3)$ \\
\hline${ }^{208} \mathrm{~Pb}\left(v_{e}, \mathrm{e}^{-} \gamma\right)^{208} \mathrm{Bi}$ & $4.7(1)$ & $1.3(2)$ & $2.5(2)$ & $4.0(2)$ & $3.5(1)$ & $7.6(1)$ & $2.2(2)$ \\
${ }^{208} \mathrm{~Pb}\left(v_{e}, \mathrm{e}^{-} \mathrm{n}\right){ }^{207} \mathrm{Bi}$ & $2.3(2)$ & $9.9(2)$ & $2.3(3)$ & $4.0(3)$ & $1.2(2)$ & $4.2(2)$ & $1.9(3)$ \\
${ }^{208} \mathrm{~Pb}\left(v_{e}, \mathrm{e}^{-} \mathrm{p}\right){ }^{207} \mathrm{~Pb}$ & $1.8(-2)$ & $1.1(-1)$ & $3.3(-1)$ & $6.9(-1)$ & $7.2(-3)$ & $3.3(-2)$ & $2.3(-1)$ \\
${ }^{208} \mathrm{~Pb}\left(v_{e}, \mathrm{e}^{-} \alpha\right){ }^{204} \mathrm{Tl}$ & $2.1(-2)$ & $2.6(-1)$ & $1.1(0)$ & $3.0(0)$ & $4.7(-3)$ & $4.1(-2)$ & $6.0(-1)$ \\
${ }^{208} \mathrm{~Pb}\left(v_{e}, \mathrm{e}^{-}\right) \mathrm{X}$ & $2.8(2)$ & $1.1(3)$ & $2.5(3)$ & $4.5(3)$ & $1.6(2)$ & $4.9(2)$ & $2.1(3)$ \\
${ }^{208} \mathrm{~Pb}\left(\bar{v}_{e}, \mathrm{e}^{+} \gamma\right){ }^{208} \mathrm{Tl}$ & $5.8(-1)$ & $3.0(0)$ & $7.9(0)$ & $1.5(1)$ & $2.7(-1)$ & $1.1(0)$ & $6.1(0)$ \\
${ }^{208} \mathrm{~Pb}\left(\bar{v}_{e}, \mathrm{e}^{+} \mathrm{n}\right){ }^{207} \mathrm{Tl}$ & $4.9(-1)$ & $3.8(0)$ & $1.5(1)$ & $3.9(1)$ & $2.0(-1)$ & $8.9(-1)$ & $8.5(0)$ \\
${ }^{208} \mathrm{~Pb}\left(\bar{v}_{e}, \mathrm{e}^{+} \mathrm{p}\right)^{207} \mathrm{Hg}$ & $1.7(-7)$ & $1.4(-5)$ & $2.2(-4)$ & $1.5(-3)$ & $8.4(-9)$ & $3.2(-7)$ & $4.2(-5)$ \\
${ }^{208} \mathrm{~Pb}\left(\bar{v}_{e}, \mathrm{e}^{+} \alpha\right){ }^{204} \mathrm{Au}$ & $4.3(-7)$ & $4.0(-5)$ & $6.5(-4)$ & $4.4(-3)$ & $2.1(-8)$ & $8.1(-7)$ & $1.2(-4)$ \\
${ }^{208} \mathrm{~Pb}\left(\bar{v}_{e}, \mathrm{e}^{+}\right) \mathrm{X}$ & $1.1(0)$ & $6.8(0)$ & $2.3(1)$ & $5.4(1)$ & $4.7(-1)$ & $1.9(0)$ & $1.5(1)$ \\
\hline
\end{tabular}

the neutron threshold is higher in even-even nuclei than in odd-A, explaining the odd-even staggering in the partial neutron decay cross sections. Again, the difference of the total cross sections compared to the sum of partial proton and neutron decay cross sections gives the $\left(\nu, v^{\prime} \gamma\right)$ cross sections, caused mainly by the Gamow-Teller strength below the particle thresholds.

We note that neutrino-induced reactions on nuclei in the iron mass region might also play a role during the collapse and the shock-revival phase of a supernova [51, 52]. Under these conditions, the cross sections have to be evaluated at the finite temperature of the stellar environment involving excited states of the parent nucleus. Relevant cross sections are presented in $[53,54]$.

\subsection{Lead}

The Fermi and Ikeda sum rules both scale with neutron excess $(N-Z)$. As the chargedcurrent response induced by supernova $v_{e}$ neutrinos (with average energies around $12 \mathrm{MeV}$ ) is dominated by Fermi and GT transitions, the charged-current cross sections on lead are expected to be significantly larger than on other materials such as iron or carbon. This makes ${ }^{208} \mathrm{~Pb}$ an attractive target for a supernova neutrino detector.

To calculate the relevant neutrino-induced cross sections on ${ }^{208} \mathrm{~Pb}$ we note that convergent shell-model calculations of the GT strength distribution are not computationally feasible. Thus, unlike in ${ }^{40} \mathrm{Ar}$ and ${ }^{56} \mathrm{Fe}$, the $\lambda^{\pi}=1^{+}$response has been evaluated within the RPA approach which fulfils the Fermi and Ikeda sum rules. As the $S_{\beta^{+}}$strength (in this direction a proton is changed into a neutron) is strongly suppressed for ${ }^{208} \mathrm{~Pb}$, the Ikeda sum rule fixes the $S_{\beta^{-}}$ strength. In the calculation the $\lambda^{\pi}=1^{+}$strength in ${ }^{208} \mathrm{~Pb}$ was renormalized by the universal quenching factor which, due to a very slight $A$-dependence is recommended to be $(0.7)^{2}$ in ${ }^{208} \mathrm{~Pb}$ [55]. Thus, the Ikeda sum rule reads in this case $S_{\beta_{-}}-S_{\beta_{+}} \approx S_{\beta_{-}}=3 \times(0.7)^{2} \times(N-Z)$. For the other multipole operators no experimental evidence exists for such a rescaling and we have used the RPA response. The RPA calculation is described in detail in [56], which also discusses the relevant neutrino-induced cross sections for ${ }^{208} \mathrm{~Pb}$ assuming a pion-decay-at-rest spectrum.

The total and partial cross sections for charged current $\left(v_{e}, \mathrm{e}^{-}\right)$and $\left(\bar{v}_{e}, \mathrm{e}^{+}\right)$reactions on ${ }^{208} \mathrm{~Pb}$ are listed in table 7 . The $\left(v_{e}, e\right)$ cross section on ${ }^{208} \mathrm{~Pb}$ is about 20 times larger than for ${ }^{56} \mathrm{Fe}$. This is caused by the $(N-Z)$ and by the strong Z-dependence of the Fermi function. 
Table 8. Total cross sections for neutral current neutrino scattering on ${ }^{208} \mathrm{~Pb}$ for different neutrino energy spectra represented as Fermi-Dirac distributions. The cross sections are in units of $10^{-42} \mathrm{~cm}^{2}$ and are averaged over neutrinos and antineutrinos.

\begin{tabular}{llllllll}
\hline$(T, \alpha)$ & $(4,0)$ & $(6,0)$ & $(8,0)$ & $(10,0)$ & $(3,-3)$ & $(4,-3)$ & $(6.26,-3)$ \\
\hline${ }^{208} \mathrm{~Pb}\left(v, v^{\prime} \gamma\right)^{208} \mathrm{~Pb}$ & $1.4(0)$ & $7.4(0)$ & $2.1(1)$ & $4.5(1)$ & $7.0(-1)$ & $2.5(0)$ & $1.5(1)$ \\
${ }^{208} \mathrm{~Pb}\left(v, v^{\prime} \mathrm{n}\right)^{207} \mathrm{~Pb}$ & $1.2(1)$ & $4.8(1)$ & $1.2(2)$ & $2.3(2)$ & $6.9(0)$ & $2.0(1)$ & $9.4(1)$ \\
${ }^{208} \mathrm{~Pb}\left(v, v^{\prime} \mathrm{p}\right)^{207} \mathrm{Tl}$ & $1.6(-5)$ & $3.5(-4)$ & $2.4(-3)$ & $8.7(-3)$ & $2.9(-6)$ & $3.1(-5)$ & $9.0(-4)$ \\
${ }^{208} \mathrm{~Pb}\left(v, v^{\prime} \alpha\right){ }^{204} \mathrm{Hg}$ & $7.8(-5)$ & $3.0(-3)$ & $2.6(-2)$ & $1.1(-1)$ & $8.1(-6)$ & $1.5(-4)$ & $7.9(-3)$ \\
${ }^{208} \mathrm{~Pb}\left(v, v^{\prime}\right) \mathrm{X}$ & $1.3(1)$ & $5.6(1)$ & $1.4(2)$ & $2.7(2)$ & $7.6(0)$ & $2.3(1)$ & $1.1(2)$ \\
\hline
\end{tabular}

Furthermore, as the IAS energy and the GT - strength are above the neutron threshold in ${ }^{208} \mathrm{Bi}$ at $6.9 \mathrm{MeV}$, most of the $\left(v_{e}, e\right)$ cross section leads to particle-unbound states and hence decays by the neutron emission. Table 8 summarizes the total and partial cross sections for neutral current reactions on ${ }^{208} \mathrm{~Pb}$.

There have been other calculations of the neutrino-induced cross sections on ${ }^{208} \mathrm{~Pb}$. The first study, performed in [36], has been criticized and improved in [57]. These authors estimated the allowed transitions to the charged-current and neutral-current cross sections empirically using data from $(\mathrm{p}, \mathrm{n})$ scattering and from the M1 response to fix the GamowTeller contributions to the cross section. Reference [57] completed their cross section estimates by calculating the first-forbidden contributions on the basis of the Goldhaber-Teller model. They calculated cross sections which are somewhat larger than the RPA results of [56]. More results have been reported in [58-60], which agree well with the RPA results. The later approach [60] is particularly interesting as it uses the experimental GT distribution, which has recently been determined in Osaka [61], and the peaks of the spin-dipole response to constrain the (Hartree-Fock + Tamm-Dancoff) calculation. Furthermore, the spreading and quenching of the GT response has been considered by coupling to $2 \mathrm{p}-2 \mathrm{~h}$ configurations. Suzuki and Sagawa obtain $\left(3.2 \times 10^{-39} \mathrm{~cm}^{2}\right)$ for the $\left(v_{e}, \mathrm{e}^{-}\right)$cross sections, assuming a DAR neutrino spectrum, in close agreement of Kolbe's RPA result $\left(3.62 \times 10^{-39} \mathrm{~cm}^{2}\right)[56]$.

Proposed detectors such as LAND and OMNIS will detect the neutrons produced by the neutrino-induced reactions on ${ }^{208} \mathrm{~Pb}$. An obvious, but not very sensitive neutrino signal is the total neutron count rate. The two detectors might also be capable of detecting the neutron energy spectrum following the decay of states in the daughter nucleus after excitation by charged- and neutral-current neutrino reactions. The expected spectra have been predicted in [56]. The neutron spectrum for the charged current reaction on ${ }^{208} \mathrm{~Pb}$ is dominated by the

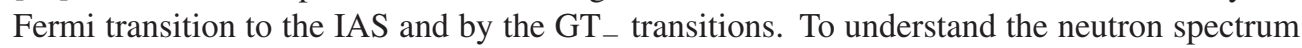
one has to consider the neutron threshold energies for one-neutron decay $(6.9 \mathrm{MeV})$ and for two-neutron decay $(14.98 \mathrm{MeV})$ in ${ }^{208} \mathrm{Bi}$. Hence the IAS and the collective GT resonance (with an excitation energy of about $16 \mathrm{MeV}$ ) will decay dominantly by $2 \mathrm{n}$ emission, while the low-lying GT - resonance at $E_{x}=8 \mathrm{MeV}$ decays by the emission of one neutron. This has significant consequences for the neutron spectrum. In the 2-neutron decay the available energy is shared between the two emitted particles, leading to a rather broad and structureless neutron energy distribution. As can be seen in figure 12 this broad structure is overlaid with a peak at neutron energy around $E_{n}=1 \mathrm{MeV}$ caused by the one-neutron decay of the lower GT - transition. One expects that, due to fragmentation which is not properly described in the RPA calculation, the width of this peak might be broader than the $0.5 \mathrm{MeV}$-binning that has been assumed in figure 12. The relative height of the peak compared to the broad structure stemming from the 2 n-emission is more pronounced for the $(T, \alpha)=(4,0)$ neutrino 


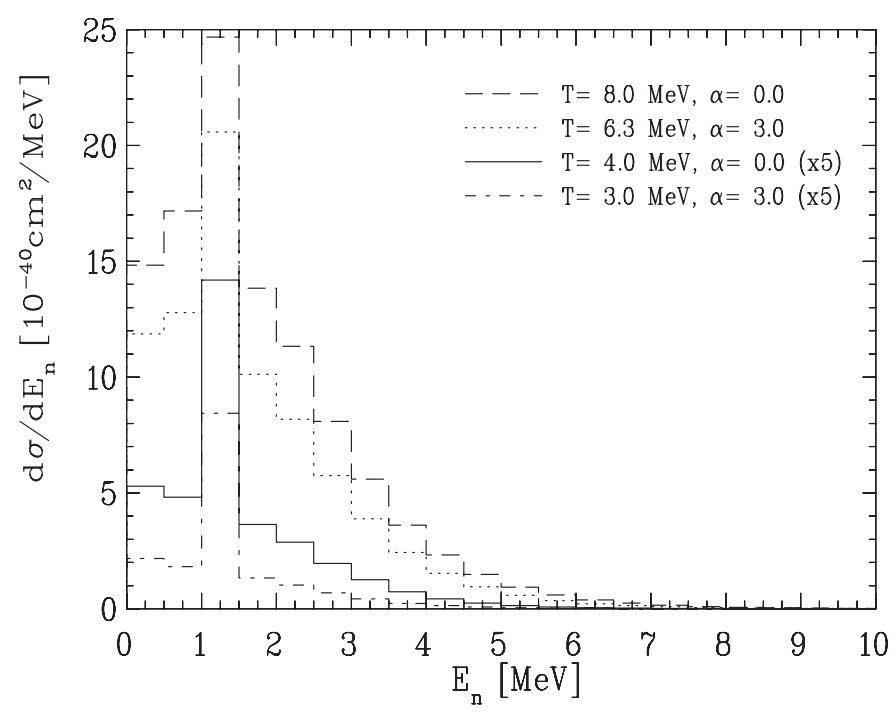

Figure 12. Neutron energy spectrum produced by the charged-current $\left(v_{e}, \mathrm{e}^{-}\right)$reaction on ${ }^{208} \mathrm{~Pb}$. The calculation has been performed for different supernova neutrino spectra characterized by the parameters $(T, \alpha)$. Note that the cross sections for $(T, \alpha)=(4,0)$ and $(3,3)$ have been scaled by a factor of 5 .

distribution than for a potential $(T, \alpha)=(8,0) v_{e}$ spectrum as it might arise after complete $v_{e} \leftrightarrow v_{\mu}$ oscillations.

Ideally, OMNIS and LAND should have the ability to detect potential neutrino oscillations. However, as has been shown in [57], the total neutron rate is by itself not suitable to detect neutrino oscillations, even if results from various detectors with different materials (hence different ratios of charged-to-neutral current cross sections, as discussed above) are combined. Reference [57] points out that in the case of ${ }^{208} \mathrm{~Pb}$ an attractive signal might emerge. Due to the fact that the IAS and large portions of the GT - strength reside in ${ }^{208} \mathrm{Bi}$ just above the 2-neutron emission threshold, Fuller et al discuss that the 2-neutron emission rate is both flavor-specific and very sensitive to the temperature of the $v_{e}$ distribution. To quantify this argument, in [56] the cross sections for the ${ }^{208} \mathrm{~Pb}\left(v_{e}, \mathrm{e}^{-2 n}\right)^{206} \mathrm{Bi}$ reaction were calculated in a model combining the RPA for the neutrino-induced response with the statistical model for the decay of the daughter states. The partial cross sections of $55.7 \times 10^{-42} \mathrm{~cm}^{2}$ for $v_{e}$ neutrinos with $(T, \alpha)=(4,0)$ Fermi-Dirac distributions should increase significantly if neutrino oscillations occur, as pointed out by [57]. For example, one finds for total $v_{e} \leftrightarrow v_{\mu}$ oscillations partial $2 \mathrm{n}$ cross sections of $1560 \times 10^{-42} \mathrm{~cm}^{2}$ (for neutrino distributions with parameters $(T, \alpha)=(8,0))$. We remark that these numbers will be probably reduced, if correlations beyond the RPA are taken into account, as part of the GT _ distribution might be shifted below the 2 n-threshold.

One has to note that this 2-neutron signal will compete with the 2-neutron decay stemming from the neutral-current reaction and hence will reduce the flavour-sensitivity of the signal. Reference [56] predicts that the combined 2n-signal resulting from neutral-current reactions for the $4 v_{x}$ neutrino types is larger than that from the charged-current reactions. However, if neutrino oscillations occur the neutral-current signal is unaffected while the charged-current signal is drastically enhanced. This supports the suggestions of [57] that the $2 n$-signal for ${ }^{208} \mathrm{~Pb}$ detectors might be an interesting neutrino oscillation signal. 


\section{Neutrino nucleosynthesis}

When the flux of neutrinos generated by the cooling of the neutron star in a type II supernova passes through the overlying shells of heavy elements, substantial nuclear transmutations are induced, despite the extremely small neutrino-nucleus cross sections. Specific nuclei (e.g. ${ }^{10,11} \mathrm{~B},{ }^{15} \mathrm{~N},{ }^{19} \mathrm{~F}$ ) might be, by a large fraction, made by this neutrino nucleosynthesis $[62,63]$. These are the product of reaction sequences induced by neutral current $\left(v, v^{\prime}\right)$ reactions on very abundant nuclei such as ${ }^{12} \mathrm{C},{ }^{16} \mathrm{O}$ or ${ }^{20} \mathrm{Ne}$. If the inelastic excitation of these nuclei proceeds to particle-unbound levels, they will decay by emission of protons or neutrons, in this way contributing to nucleosynthesis. As the nucleon thresholds are relatively high, effectively only $v_{\mu}, v_{\tau}$ neutrinos and their antiparticles with their higher average energies contribute to the neutrino nucleosynthesis of these elements. It has been noted that the neutrino induced nucleosynthesis, the so-called $\nu$-process, might also be responsible for the production of ${ }^{138} \mathrm{La}$ and to a fraction of the ${ }^{180} \mathrm{Ta}$ abundance [62]. The ${ }^{138} \mathrm{La}$ nuclide is of special interest as it appears to be produced by the charged current $\left(v_{e}, \mathrm{e}^{-}\right)$reaction on the $s$-process element ${ }^{138} \mathrm{Ba}$, as has already been proposed in $[62,64]$ and recently been confirmed in detailed studies by Heger et al [66]. This finding is quite welcome as it makes the $v$ process sensitive to the flux and distribution of supernova $v_{e}$ and $v_{\mu}, v_{\tau}$, complementing the constraints for supernova $\bar{\nu}_{e}$ neutrinos from their observation in the water Čerenkov detectors for SN1987A. Neutrino nucleosynthesis is thus potentially an important test for the predictions of supernova models. This test is particularly stringent, if neutrino oscillations involving $v_{e}$ neutrinos occur in the supernova environment.

Neutrino nucleosynthesis has been proposed by Woosley et al [62] who also performed detailed production studies in a $20 M_{\odot}$ star. Later Timmes et al have extended this calculation to studies of the $v$ process in a full galactical model [63]. Very recently, Heger et al [66] have improved these earlier studies by considering mass loss in the evolution of the progenitor stars, and by using a complete and updated reaction network which includes all the heavy elements through bismuth. This improvement is essential for a consistent treatment of the s-process in the progenitor star. Last, but not the least the studies of [66] also used improved neutrino-induced reaction cross sections for the key nuclei of the $v$ process. For the $p$ and $s d$ shell nuclei the allowed neutrino response has been evaluated on the basis of the shell model, while the forbidden responses were calculated within the framework of the RPA. For the heavier nuclei, in particular for the progenitors of ${ }^{138} \mathrm{La}$ and ${ }^{180} \mathrm{Ta}$, all cross sections were derived within the RPA. The various partial decay cross sections were then evaluated using a statistical model cascade.

As stressed above, Heger et al [66] followed the neutrino nucleosynthesis of the light (e.g. ${ }^{11} \mathrm{~B},{ }^{19} \mathrm{~F}$ ) and heavy (e.g. ${ }^{138} \mathrm{La},{ }^{180} \mathrm{Ta}$ ) candidate nuclei in a self-consistent way in complete stellar evolution models that included the evolution of all isotopes up to bismuth from the time the star ignited central hydrogen burning through the supernova explosion. A consistent stellar modelling, including a sufficient nuclear network, is important for two reasons. At first, it is essential to reliably describe the production of the progenitor nuclides $\left(\right.$ e.g. ${ }^{12} \mathrm{C},{ }^{20} \mathrm{Ne}$, ${ }^{138} \mathrm{Ba}$, etc) in the presupernova evolution. Secondly, the nuclides produced by neutrinos from these progenitors can suffer severe photo-dissociations due to heating by the supernova shock wave which passes the region of neutrino nucleosynthesis after the neutrinos. The neutrino production factors for four key nuclides are summarized in figure 14 for $15 M_{\odot}$ and $25 M_{\odot}$ stars. The results are shown relative to the ${ }^{16} \mathrm{O}$ production in these stars recognizing that the solar ${ }^{16} \mathrm{O}$ abundance is an indicator for core-collapse supernova element production.

The calculation [66] confirms earlier studies $[62,63]$ that the neutrino nucleosynthesis makes a large fraction of the solar ${ }^{11} \mathrm{~B}$ and ${ }^{19} \mathrm{~F}$ abundance. For both nuclides, neutral-current 


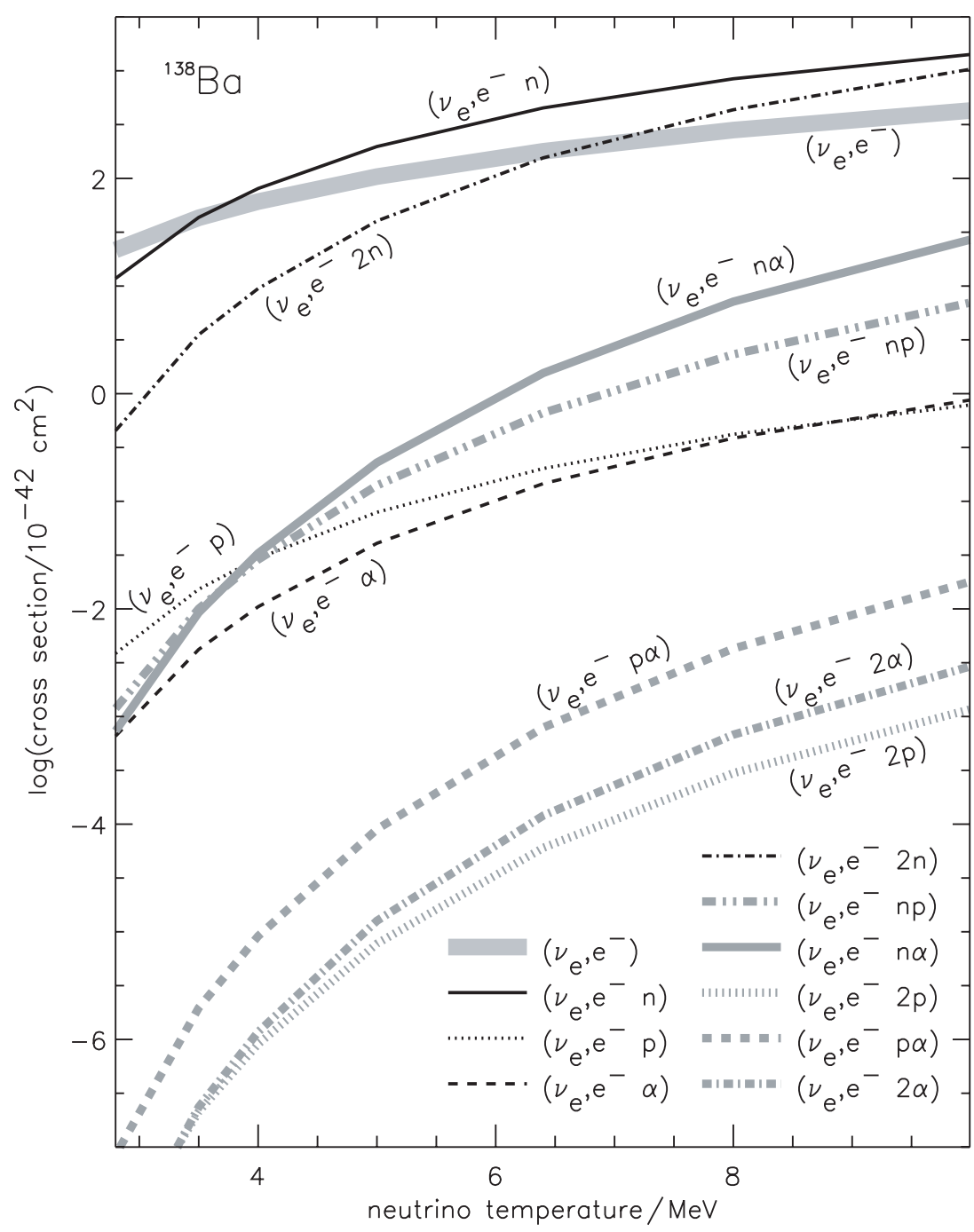

Figure 13. Partial cross sections for the $v_{e}$-induced charged-current reaction on ${ }^{138} \mathrm{Ba}$. The calculations were performed for Fermi-Dirac neutrino spectra with $\alpha=0$ and different temperature values (from [66]).

reactions, on ${ }^{12} \mathrm{C}$ and ${ }^{20} \mathrm{Ne}$, induced by $v_{x}$ neutrinos are the main production source. The new calculation predicts a somewhat smaller production of ${ }^{19} \mathrm{~F}$ than the earlier studies. A measurement of the $\mathrm{GT}_{0}$ response on ${ }^{20} \mathrm{Ne}$ might be quite desirable, including the cascade of decays of excited states, to better constrain the $\left(v, v^{\prime}\right)$ cross section on ${ }^{20} \mathrm{Ne}$. As predicted in $[62,64],{ }^{138} \mathrm{La}$ is mainly produced by charged-current reactions on ${ }^{138} \mathrm{Ba}$ (see figure 13 ), while the $\gamma$-process contribution is small and the neutral-current contribution from ${ }^{139} \mathrm{La}$, which had been speculated to be the possible ${ }^{138}$ La production process via $\left(\nu, v^{\prime} n\right)$, is insignificant. One observes that enough ${ }^{138} \mathrm{La}$ is being made to explain the solar abundance, where the $s$-process production of the parent ${ }^{138} \mathrm{Ba}$ in the $s$-process prior to the supernova plays an essential role. The calculations also show a significant production of the rarest nuclide ${ }^{180} \mathrm{Ta}$, where both, charged- and neutral-current processes contribute. The ${ }^{180}$ Ta production factors 


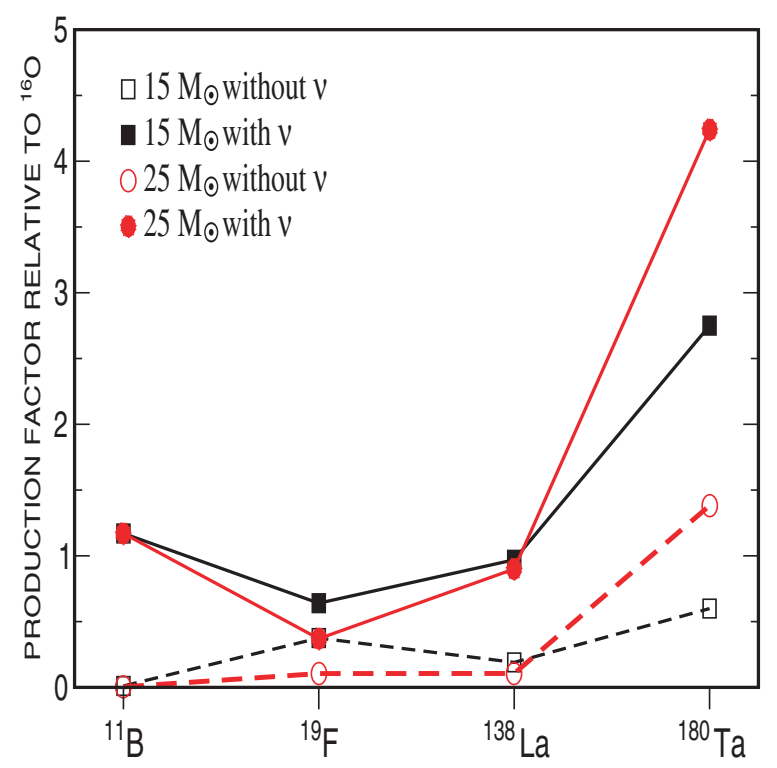

Figure 14. Production factor of ${ }^{11} \mathrm{~B},{ }^{19} \mathrm{~F},{ }^{138} \mathrm{La}$ and ${ }^{180} \mathrm{Ta}$ relative to ${ }^{16} \mathrm{O}$ in $15 M_{\odot}$ (squares) and $25 M_{\odot}$ (circles) stars (from [66]). The open (filled) symbols represent stellar evolution studies in which neutrino reactions on nuclei were excluded (included).

in figure 14 add the contributions of the ground state and the $9^{-}$isomeric state. As the ground state is rather short-lived $(\sim 4.5 \mathrm{~h})$, the isomeric state is the sole contributor to the solar ${ }^{180} \mathrm{Ta}$ abundance. Estimates [65] indicate that at thermal freeze-out about $30-50 \%$ of ${ }^{180} \mathrm{Ta}$ is in the isomeric state bringing the production factors of figure 14 in closer agreement with the solar abundances.

Importantly the ${ }^{138} \mathrm{La}$ and ${ }^{180} \mathrm{Ta}$ production is sensitive to the neutrino distributions. For example, if the temperature of the $v_{e}$ spectrum increases from $4 \mathrm{MeV}$ to $6 \mathrm{MeV}$ (which might correspond to a neutrino oscillation scenario) the ${ }^{138}$ La production factor increases by a factor 2 and, in the study of [66], this nuclide would already be overproduced. This makes the neutrino nucleosynthesis of ${ }^{138} \mathrm{La}$ a potentially interesting test for neutrino oscillations, in particular for the yet unmeasured neutrino mixing angle $\theta_{13}$ [66].

Finally, we note that $v$ nucleosynthesis of ${ }^{138} \mathrm{La}$ and ${ }^{180} \mathrm{Ta}$ competes with the p-process production of these elements, making a reliable determination of the p-process abundances also important.

\section{Conclusions}

In this review we have described the methods used in the evaluation of the neutrino-nucleus reaction cross sections. We have shown, in detail, the results of calculations of these cross sections for a variety of targets of practical importance.

Many of the described reactions are accessible in experiments with a neutrino source from the pion and muon decay at rest, available at the future very intense neutron spallation sources. Detailed comparison of the results of such experiments would establish important benchmarks for comparison of theory and experiment. This, in turn, would lead to refinements or possible modifications of the theoretical treatment of processes involving the charged and 
neutral current interaction of neutrinos with complex nuclei. Having a reliable tool for such calculation is of great importance in a variety of applications, such as the study of neutrino oscillations, detection of supernova neutrino signal, description of the neutrino transport in supernovae, or description of the $r$-process nucleosynthesis.

\section{Acknowledgments}

The work of PV was supported in part by the US DOE and by the Institute of Physics and Astronomy at the University of Århus. KL is partially supported by the Danish Research Foundation. GMP is supported by the Spanish MCyT under contracts AYA2002-04094-C0302 and AYA2003-06128.

\section{References}

[1] Walecka J D 1972 Semi-leptonic weak interactions in nuclei Muon Physics ed V W Hughes and C S Wu (New York: Academic)

[2] O’Connell J S, Donnelly T W and Walecka J D 1972 Phys. Rev. C 6719

[3] Donnelly T W and Haxton W C 1979 At. Data Nucl. Data. Tables 23103

[4] Donnelly T W and Peccei R D 1979 Phys. Rep. 501

[5] Kolbe E 1996 Phys. Rev. C 541741

[6] Behrens H and Bühring W 1982 Electron Radial Wave Functions and Nuclear Beta-Decay (Oxford: Clarendon)

[7] Engel J 1998 Phys Rev. C 572004

[8] Caurier E, Martinez-Pinedo G, Nowacki F, Poves A and Zuker A Rev. Mod. Phys. at press

[9] Buballa M, Drozdz S, Krewald S and Speth J 1991 Ann. Phys., NY 208346

[10] Kolbe E, Langanke K, Krewald S and Thielemann F-K 1992 Nucl. Phys. A 540599

[11] Smith R A and Moniz E J 1972 Nucl. Phys. B 43605

[12] Cowan J J, Thielemann F K and Truran J W 1991 Phys. Rep. 208208 Rauscher T and Thielemann F-K 2000 At. Data Nucl. Data Tables 751 Rauscher T and Thielemann F-K 2001 At. Data Nucl. Data Tables 7947

[13] Langanke K, Vogel P and Kolbe E 1996 Phys. Rev. Lett. 762629

[14] Haxton W C 1987 Phys. Rev. D 362283

[15] Kolbe E, Langanke K and Vogel P 1994 Phys. Rev. C 502576

[16] Drexlin G et al (KARMEN Collaboration) 1991 Phys. Lett. B 267321 Zeitnitz B (KARMEN Collaboration) 1994 Prog. Part. Nucl. Phys. 32351 Kleinfeler J et al (KARMEN Collaboration) 1997 Neutrino'96 ed K Enquist, K Huitu and J Maalampi (Singapore: World Scientific)

[17] Albert M et al 1995 Phys. Rev. C 511065 Athanassopoulos C et al 1997 Phys. Rev. C 552078 Athanassopoulos C et al 1997 Phys. Rev. C 562806 Imlay R 1998 Nucl. Phys. A $629531 \mathrm{c}$

[18] Krakauer D A et al 1992 Phys. Rev. C 452450

[19] Kolbe E, Langanke K and Krewald S 1994 Phys. Rev. C 491122

[20] Kolbe E, Langanke K, Thielemann F-K and Vogel P 1995 Phys. Rev. C 523437

[21] Kolbe E, Langanke K and Vogel P 1999 Nucl. Phys. A 65291

[22] Kosmas T S and Oset E 1996 Phys. Rev. C 531409

[23] Singh S K, Mukhopadhyay N C and Oset E 1998 Phys. Rev. C 572687

[24] Mintz S L and Pourkaviani M 1995 Nucl. Phys. A 594346

[25] Auerbach N, Van Giai N and Vorov O K 1997 Phys. Rev. C 56 R2368

[26] Engel J, Kolbe E, Langanke K and Vogel P 1996 Phys. Rev. C 542740

[27] Fukugita M, Kohyama Y and Kubodera K 1988 Phys. Lett. B 212139

[28] Leiss J E and Taylor R E, as quoted in Czyz W 1963 Phys. Rev. 1312141

[29] Kolbe E, Langanke K and Vogel P 1996 Nucl. Phys. A 613382

[30] Hayes A C and Towner I S 2001 Phys. Rev. C 610044604

[31] Auerbach N and Brown B A 2002 Phys. Rev. C 65024322

[32] Raffelt G 1996 Stars as Laboratories for Fundamental Physics (Chicago, IL: University of Chicago Press) 
[33] Hirata K S et al 1987 Phys. Rev. Lett. 581490

[34] Bionta R M et al 1987 Phys. Rev. Lett. 581494

[35] Cline D B et al 1990 Astrophys. Lett. Commun. 27403

Cline D B et al 1994 Phys. Rev. D 50720

[36] Hargrove C K et al 1996 Astroparticle Phys. 5183

[37] Ewan G 1992 Nucl. Instr. and Methods A 314373

SNO Collaboration 1992 Phys. Canada 48112

[38] Th Keil M, Raffelt G and Janka H-T 2002 Preprint astro-ph/0208035

[39] Janka H-T and Hillebrandt W 1989 Astron. Astrophys. 22449

Janka H-T and Hillebrandt W 1989 Astron. Astrophys. Suppl. 78375

[40] Wilson J R (private communication), as cited in Qian Y-Z, Haxton W C, Langanke K and Vogel P 1997 Phys. Rev. C 551532

[41] Kolbe E, Langanke K and Martinez-Pinedo G 1999 Phys. Rev. C 60052801

[42] Hektor A, Kolbe E, Langanke K and Toivanen J 2000 Phys. Rev. C 61055803

[43] Totsuka Y 1992 Rep. Prog. Phys. 55377

[44] Nussinov S and Shrock R 2001 Phys. Rev. Lett. 862223

[45] Kolbe E, Langanke K and Vogel P 2002 Phys. Rev. D 66013007

[46] Engel J, Kolbe E, Langanke K and Vogel P 1993 Phys. Rev. C 483048

[47] Cavanna F and Palamara P 2002 Nucl. Phys. B 112265

[48] Ormand W E, Pizzochero P M, Bortignon P F and Broglia R A 1995 Phys. Lett. B 345343

[49] Maschuw R 1998 Prog. Part. Phys. 40183

Eitel K 1998 Private communication

[50] Toivanen J, Kolbe E, Langanke K, Martinez-Pinedo G and Vogel P 2001 Nucl. Phys. A 694395

[51] Haxton W C 1988 Phys. Rev. Lett. 601999

[52] Langanke K and Martinez-Pinedo G 2003 Rev. Mod. Phys. 75819

[53] Langanke K, Martinez-Pinedo G and Sampaio J M 2001 Phys. Rev. C 64055801

[54] Sampaio J M, Langanke K, Martinez-Pinedo G and Dean D J 2002 Phys. Lett. B 52919

[55] Martinez-Pinedo G, Poves A, Caurier E and Zuker A P 1996 Phys. Rev. C 53 R2602

[56] Kolbe E and Langanke K 2001 Phys. Rev. C 63025802

[57] Fuller G M, Haxton W C and McLaughlin G C 1999 Phys. Rev. D 59085005

[58] Jachowitz N, Heyde K and Ryckebush J 2000 Phys. Rev. C 66055501

[59] Volpe C, Aurbach N, Colo G and Van Giai N 2002 Phys. Rev. C 65044603

Engel J, McLaughlin G C and Volpe C 2003 Phys. Rev. C 67013005

[60] Suzuki T and Sagawa H 2003 Nucl. Phys. A 718 446c

[61] Krasznahorkay A et al 2001 Phys. Rev. C 64067302

[62] Woosley S E, Hartmann D H, Hoffman R D and Haxton W C 1990 Astron. J. 356272

[63] Timmes F X, Woosley S E and Weaver T A 1995 Astrophys. J. Suppl. 98617

[64] Goriely S, Arnould M, Borsov I and Rayet M 2001 Astron. Astrophys. 37535

[65] Rauscher T, Heger A, Hoffman R D and Woosley S E 2002 Astrophys. J. 576323

[66] Heger A, Kolbe E, Haxton W C, Langanke K, Martinez-Pinedo G and Woosley S E submitted to PRL 